ARTICLE

Received 27 Aug 2015 | Accepted 8 Jun 2016 | Published 14 Jul 2016

DOI: $10.1038 /$ ncomms12180

OPEN

\title{
SREBP1c-CRY1 signalling represses hepatic glucose production by promoting FOXO1 degradation during refeeding
}

Hagoon Jang ${ }^{1}$, Gha Young Lee ${ }^{1}$, Christopher P. Selby², Gung Lee ${ }^{1}$, Yong Geun Jeon', Jae Ho Lee ${ }^{1}$, Kenneth King Yip Cheng ${ }^{3}$, Paul Titchenell ${ }^{4}$, Morris J. Birnbaum ${ }^{4}$, Aimin Xu ${ }^{3}$, Aziz Sancar ${ }^{2}$ \& Jae Bum Kim ${ }^{1}$

SREBP1c is a key lipogenic transcription factor activated by insulin in the postprandial state. Although SREBP1c appears to be involved in suppression of hepatic gluconeogenesis, the molecular mechanism is not thoroughly understood. Here we show that CRY1 is activated by insulin-induced SREBP1c and decreases hepatic gluconeogenesis through FOXO1 degradation, at least, at specific circadian time points. SREBP1C ${ }^{-/-}$and $\mathrm{CRY}^{-/-}$mice show higher blood glucose than wild-type (WT) mice in pyruvate tolerance tests, accompanied with enhanced expression of PEPCK and G6Pase genes. CRY1 promotes degradation of nuclear FOXO1 by promoting its binding to the ubiquitin E3 ligase MDM2. Although SREBP1c fails to upregulate CRY1 expression in $d b / d b$ mice, overexpression of CRY1 attenuates hyperglycaemia through reduction of hepatic FOXO1 protein and gluconeogenic gene expression. These data suggest that insulin-activated SREBP1c downregulates gluconeogenesis through CRY1-mediated FOXO1 degradation and that dysregulation of hepatic SREBP1C-CRY1 signalling may contribute to hyperglycaemia in diabetic animals.

\footnotetext{
${ }^{1}$ School of Biological Sciences, Institute of Molecular Biology and Genetics, Center for Adipose Tissue Remodeling, Seoul National University, Seoul 151-742, Korea. ${ }^{2}$ Department of Biochemistry and Biophysics, University of North Carolina School of Medicine, CB \# 7260, Chapel Hill, North Carolina 27599-7260, USA. ${ }^{3}$ State Key Laboratory of Pharmaceutical Biotechnology and Department of Medicine, The University of Hong Kong, Hong Kong, 999077, China. ${ }^{4}$ The Institute for Diabetes, Obesity and Metabolism, Perelman School of Medicine, University of Pennsylvania, Philadelphia, Pennsylvania 19104, USA.

Correspondence and requests for materials should be addressed to J.B.K. (email: jaebkim@snu.ac.kr).
} 
nsulin, which is released from pancreatic $\beta$-cells, plays a key role in the maintenance of the whole body energy homoeostasis by actively regulating glucose and lipid metabolism. In the postprandial state, insulin lowers blood glucose by stimulating glucose uptake in adipose tissues and muscles as well as by inhibiting hepatic glucose production ${ }^{1,2}$. Moreover, in the liver, insulin stimulates the conversion of excess glucose into glycogen (glycogenesis) and triacylglyceride (lipogenesis) for the long-term energy storage ${ }^{3-5}$.

Suppression of hepatic gluconeogenesis by insulin is an important process to inhibit hyperglycaemia. PEPCK and G6Pase are crucial enzymes that convert pyruvate to glucose, and their gene expression is regulated by several transcription factors such as Forkhead box O1 (FOXO1), cAMP response elementbinding protein (CREB), hepatocyte nuclear factor 4 (HNF4), glucocorticoid receptor and peroxisome proliferator-activated receptor gamma coactivator 1 -alpha $(\mathrm{PGC1} \alpha)^{6-8}$. In the liver, FOXO1 is activated on fasting and gets inactivated by feeding, which is one of the essential mechanisms by which insulin rapidly and efficiently represses hepatic glucose production during postprandial periods ${ }^{9-11}$. After insulin treatment, FOXO1 protein is phosphorylated by AKT and then moves to the cytoplasm, resulting in the decrease of gluconeogenic gene expression $^{12}$. Although the translocation of hepatic FOXO1 from the nucleus to the cytoplasm is a well-defined mechanism mediating a quick decrease in glucose production by insulin, it is largely unknown how insulin endows a sustainable inhibition of hepatic gluconeogenesis throughout the postprandial state.

On the other hand, SREBP1c has been proposed to be involved in the regulation of hepatic glucose metabolism. SREBP1c is a basic-helix-loop-helix-leucine zipper (bHLH-LZ) transcription factor that regulates de novo lipogenesis ${ }^{13-17}$. Activation of SREBP1c is mediated by AKT and mTORC1 on insulin signalling ${ }^{18,19}$. SREBP1c regulates lipogenic pathways by stimulating the expression of target genes such as those encoding fatty acid synthase (FASN), stearoyl-CoA desaturase 1 $(S C D 1)$, and acetyl-coenzyme $A$ carboxylase $(A C C)^{20-22}$. In addition, SREBP1c appears to regulate hepatic carbohydrate metabolism. For example, SREBP1c affects the mRNA levels of $P E P C K$, G6Pase and IRS-2 genes and inhibits the interaction between HNF4 and PGC1 $\alpha$ to suppress gluconeogenic genes ${ }^{23-27}$. Although hepatic SREBP1c has been reported to be upregulated in obese animals, the reason why increased SREBP1c fails to repress hepatic gluconeogenesis is unknown. Thus, understanding the molecular mechanisms by which SREBP1c could modulate gluconeogenesis under physiological and pathological conditions is important.

CRY1 is a member of the mammalian clock genes regulated by transcription-translation feedback loop that also includes CLOCK, BMAL1, PER1, PER2 and CRY2, to modulate rhythmic oscillations. CLOCK and BMAL1 form a heterodimer to activate $P E R$ and $C R Y$ genes and then elevated PER and CRY proteins act as transcriptional repressors that decrease the transcriptional activity of CLOCK and BMAL1 (refs 28-31). The hepatic circadian clock is regulated by food intake and by the expression of hormones such as insulin and glucagon, whereas the suprachiasmatic nucleus circadian clock is controlled by the light-dark cycle ${ }^{32}$. Recently, it has been shown that hepatic circadian clock genes also contribute to glucose homoeostasis. For example, hepatic CRY proteins modulate glucose production by inhibiting the glucagon receptor signalling pathway and binding to glucocorticoid receptor ${ }^{33,34}$. In addition, an agonist of CRY proteins has been reported to repress the expression of hepatic gluconeogenic genes, such as PEPCK and G6Pase ${ }^{35}$. Furthermore, BMAL1 $1^{-}$mice exhibited disrupted hepatic glucose homoeostasis ${ }^{36}$. However, the molecular mechanisms by which
CRY1 could repress hepatic glucose production during the postprandial state remain to be elucidated.

The fact that SREBP1c downregulates hepatic gluconeogenesis prompted us to investigate novel target genes of SREBP1c by comparing $S R E B P 1 c^{+/+}$and $S R E B P 1 c^{-/-}$mice. Here we demonstrate that SREBP1c attenuates hepatic glucose production via the activation of CRY1 at certain circadian time points (particularly at ZT 3), eventually leading to degradation of the FOXO1 protein on insulin signalling. While hepatic FOXO1 is rapidly translocated from the nucleus into the cytoplasm by AKTmediated phosphorylation triggered by insulin, the SREBP1cCRY1 signalling pathway durably represses the execution of gluconeogenic genes by decreasing nuclear FOXO1 protein for long-term insulin action. In addition, in the liver of diabetic $d b / d b$ mice, overexpression of CRY1 lowers blood glucose, accompanied with attenuated gluconeogenic gene expression and FOXO1 protein. Altogether, our data suggest that insulin activates the SREBP1c-CRY1 signalling pathway, resulting in FOXO1 degradation mediated by MDM2, which is one of the crucial mechanisms to maintain hepatic glucose homoeostasis.

\section{Results}

CRY1 is upregulated by feeding and insulin. In the liver, peripheral circadian clock genes are regulated by various nutritional and hormonal changes ${ }^{37}$. Given that circadian clock genes are closely associated with energy homoeostasis, we sought to investigate which circadian clock genes could affect feedingdependent hepatic lipid and glucose metabolism. Since the expression of most circadian clock genes oscillates in a time dependent manner, the end points of the fasting and/or refeeding experiment were fixed at ZT 3. On refeeding, the expression of lipogenic genes including SREBP1c, FASN and ACC was upregulated in the liver. In accordance with previous reports ${ }^{38-40}$, the expression of gluconeogenic genes such as PEPCK and G6Pase was downregulated in the postprandial state (Fig. 1a, Supplementary Fig. 1A). Interestingly, the level of hepatic CRY1 mRNA was elevated in refed mice (Fig. 1a). Moreover, the expression of the CRY1 protein was markedly increased in the liver of refed mice at ZT 3 (Fig. 1b, Supplementary Fig. 1B). Because CRY1 is one of core circadian clock genes, we investigated the expression of CRY1 gene at ZT 22 and ZT 10, which are the peak and trough time point of CRY1 expression, respectively ${ }^{31}$. On refeeding, the expression of many circadian clock genes including CRY1, BMAL1, CLOCK, PER1, PER2 and Reverbs was significantly changed at ZT 22, but mostly not at ZT 10 (Supplementary Fig. 2). While basal levels of CRY1 expression were differently regulated at ZT 22 and $\mathrm{ZT} 10$, refeeding increased CRY1 mRNA and protein levels at ZT 22, compared with ZT 10. These results imply that CRY1 expression would be modulated by nutritional stimuli at certain ZT time points (Supplementary Fig. 2). These findings prompted us to test whether insulin might elevate hepatic $C R Y 1$ gene expression. In primary hepatocytes, the level of CRY1 mRNA was increased by insulin, similar to SREBP1C mRNA (Fig. 1c). Altogether, these data propose that hepatic CRY1 expression would be upregulated by feeding and insulin.

SREBP1c regulates CRY1 gene expression. To investigate which transcription factors regulate insulin-dependent CRY1 gene expression, we analysed CRY1 promoters in several species including monkey, cow, sheep, human, rat and mouse (Supplementary Fig. 3A). In the proximal CRY1 promoter, there are several sterol regulatory element (SRE) motifs as well as an E-BOX (CANNTG) motif, which is also a target motif for BMAL1 and CLOCK, the core circadian clock proteins 

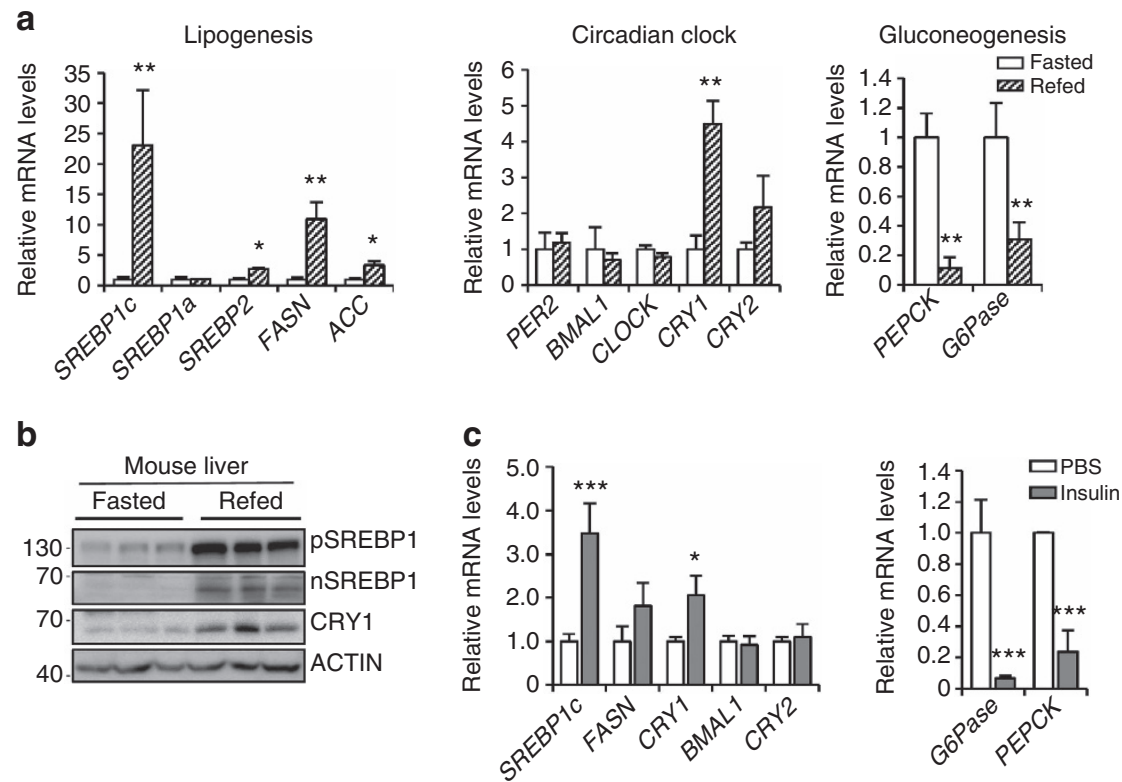

\begin{abstract}
Figure 1 | CRY1 is stimulated by feeding and exposure to insulin. (a,b) C57BL/6 mice were fasted for $24 \mathrm{~h}$ and then refed for $12 \mathrm{~h}$. Both fasted and refed mice were sacrificed at ZT 3. In the liver, levels of the CRY1 mRNA (a) and CRY1 protein (b) were determined using qRT-PCR with normalization to TBP mRNA levels and western blotting, respectively. pSREBP1, precursor SREBP1; nSREBP1, nuclear SREBP1. Data are represented as mean $\pm \mathrm{s}$.d., $N=3$ for each group. ${ }^{\star} P<0.05,{ }^{\star \star} P<0.01$ (Student's $t$-test). (c) $C R Y 1$ gene expression was measured in mouse primary hepatocytes following $12 \mathrm{~h}$ of insulin exposure using qRT-PCR. The level of the TBP mRNA was used for the qRT-PCR normalization. Data are represented as mean $\pm \mathrm{s} . \mathrm{d}$., $N=3$ for each group. ${ }^{\star} P<0.05,{ }^{\star \star \star} P<0.001$ (Student's $t$-test). See Supplementary Fig. 13 for original full immunoblot.
\end{abstract}

(Supplementary Fig. 3A). Both SRE and E-BOX motifs are well-known binding targets of SREBP1c with its dual DNA binding specificity ${ }^{41,42}$. To examine whether SREBP1c could regulate CRY1 gene expression, SREBP1c was overexpressed in mouse primary hepatocytes. As shown in Fig. 2a,b, the levels of hepatic CRY1 mRNA and CRY1 protein were increased by SREBP1c overexpression (Fig. 2a,b, Supplementary Fig. 4A), implying that SREBP1c may be a key transcription factor that upregulates hepatic CRY1 gene expression in the postprandial state. Next, the effect of ectopic expression of SREBP1c on the CRY1 promoter activity was examined. Expression of luciferase from a wild-type CRY1 promoter was compared with the expression from a promoter with mutated SRE motifs (3XSRE) in HEK293T cells (Fig. 2c). We observed substantial loss of promoter activity with loss of the 3 XSRE sequences but not E-BOX motif sequences (Fig. 2c, Supplementary Fig. 3B). In addition, SREBP1c binding to the CRY1 promoter was confirmed by a ChIP assay (Fig. 2d). Meanwhile, consistent with previous reports $^{23-25}$, hepatic SREBP1c reduced G6Pase and PEPCK expression (Fig. 2a).

To investigate whether SREBP1c might promote CRY1 gene expression on insulin, primary hepatocytes isolated from $S R E B P 1 c^{+/+}$and SREBP $1 c^{-/-}$mice were challenged with insulin. The level of CRY1 protein in SREBP $1 c^{+/+}$primary hepatocytes was prominently elevated by insulin whereas that of CRY1 protein in SREBP $1 c^{-/-}$primary hepatocytes was increased to a lesser extent by insulin (Fig. 2e). Moreover, in contrast to SREBP $1 c^{+1+}$ mice, refeeding failed to increase hepatic CRY1 gene expression in SREBP1c-/- mice (Fig. 2f). These data indicate that SREBP1c is a key factor for the upregulation of hepatic $C R Y 1$ gene expression in the postprandial state.

SREBP1c-CRY1 pathway inhibits hepatic gluconeogenesis. Consistent with previous reports ${ }^{25,33,34}$, SREBP1c overexpression decreased glucose production in mouse primary hepatocytes
(Fig. 3a). In addition, the optical in vivo imaging analysis revealed that hepatic SREBP1c overexpression remarkably repressed the promoter activity of the G6Pase gene in vivo (Fig. 3b). Accordingly, the expression of G6Pase and PEPCK genes was suppressed in the liver of SREBP1c overexpressing mice (Supplementary Fig. 4B). These findings led us to investigate the effect of SREBP1c on blood glucose level in vivo. Pyruvate tolerance test revealed that the adenoviral overexpression of SREBP1c significantly decreased the level of blood glucose following pyruvate injection (Fig. 3c,d). Also, SREBP1c $c^{-/-}$ mice showed higher blood glucose than $S R E B P 1 c^{+/+}$mice (Fig. 3e,f). Thus, these results suggest that hepatic SREBP1c would suppress gluconeogenesis, potentially by modulating gluconeogenic gene expression.

To examine whether CRY1, a novel target gene of SREBP1c, might modulate hepatic gluconeogenic gene expression, we suppressed CRY1 expression via small interfering RNA (siRNA) in rat hepatoma H4IIE cells. Downregulation of CRY1 increased the expression of G6Pase and PEPCK genes (Fig. 3g, Supplementary Fig. 5A), which are crucial for hepatic gluconeogenesis. On the contrary, hepatic CRY1 overexpression decreased the expression of G6Pase and PEPCK genes in mouse primary hepatocytes (Fig. 3h, Supplementary Fig. 5B). To confirm these observations, we measured pyruvate-induced blood glucose level from $C R Y 1^{+1+}$ and $C R Y 1^{-/-}$mice. As shown in Fig. 3i,j, $C R Y 1^{-/-}$mice exhibited higher blood glucose level than $C R Y 1^{+/+}$mice. To test whether CRY1 would be a key mediator of the inhibition of hepatic gluconeogenesis by SREBP1c, glucose production assays were performed in primary hepatocytes from $C R Y 1^{+/+}$and $C R Y 1^{-/-}$mice. As indicated in Fig. 3k and Supplementary Fig. 5C, SREBP1c overexpression attenuated glucose production in $C R Y 1^{+/+}$primary hepatocytes while SREBP1c failed to suppress glucose production in $C R Y 1^{-/-}$ primary hepatocytes. To establish whether the SREBP1c-CRY1 signalling pathway could indeed repress hepatic glucose production in vivo, CRY1 was adenovirally overexpressed in the liver of 
a

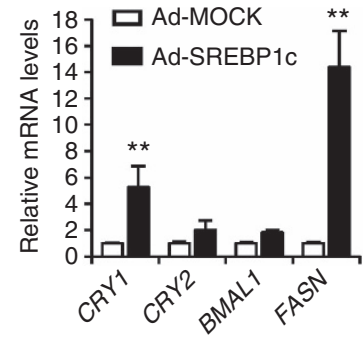

C

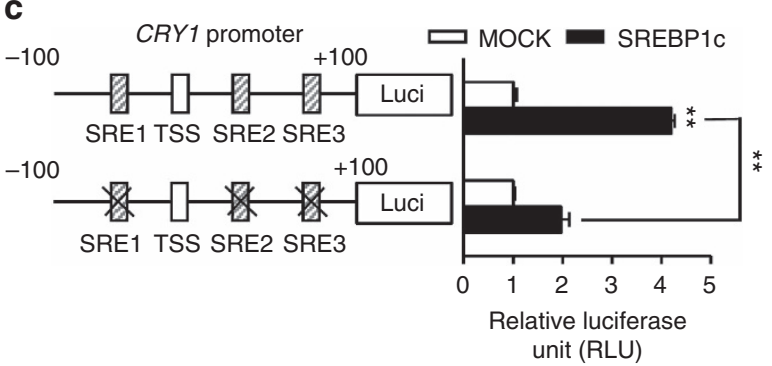

e

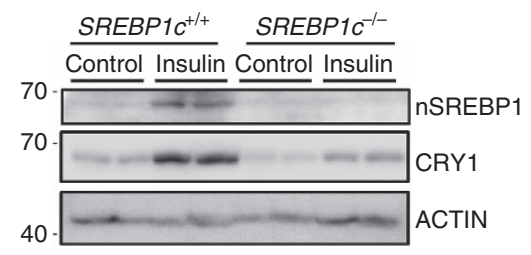

b
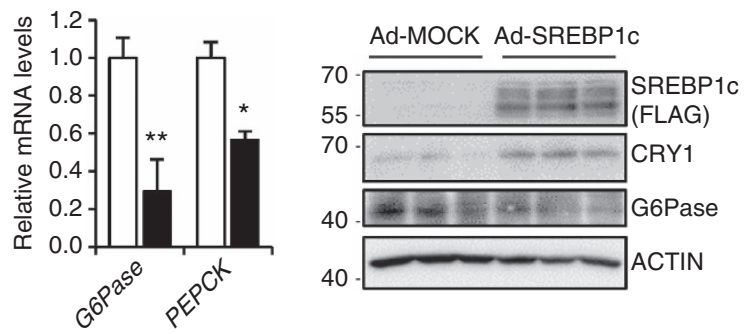
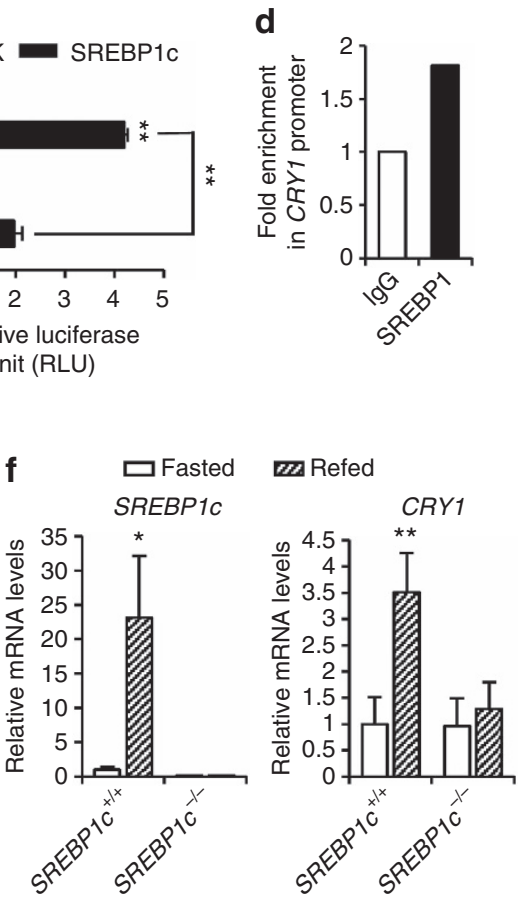

Figure 2 | SREBP1c directly activates CRY1 gene expression. (a,b) Mouse primary hepatocytes were adenovirally infected with Ad-MOCK or Ad-SREBP1C, as indicated. The levels of the CRY1 mRNA (a) and CRY1 protein (b) were determined using qRT-PCR with normalization to TBP mRNA levels and western blotting, respectively. Data are represented as mean \pm s.d., $N=3$ for each group. ${ }^{\star} P<0.05,{ }^{\star \star} P<0.01$ (Student's $t$-test). (c) Luciferase activity of the WT CRY1 promoter and 3XSRE mutant promoter were measured following co-transfection with expression plasmids encoding either SREBP1c or MOCK in HEK293T cells. Luciferase activity was normalized by $\beta$-gal activity. TSS, Transcription Start Site; SRE, Sterol Regulatory Elements. Data are represented as mean \pm s.d., $N=5$ for each group. ${ }^{\star \star} P<0.01$ (Student's $t$-test). (d) ChIP assay, performed as described in Methods section, showing CRY1 promoter occupancy by SREBP1 in H4IIE cells. (e) Mouse primary hepatocytes were isolated from SREBP1c ${ }^{-/-}$and SREBP1c $+/+$mice. With insulin (10 nM), the levels of SREBP1c and CRY1 protein were determined using western blotting. (f) SREBP1 $\mathrm{c}^{-/-}$and SREBP1c $\mathrm{c}^{+/+}$mice were fasted for $24 \mathrm{~h}$ and then refed for $12 \mathrm{~h}$. Both fasted and refed mice were sacrificed at ZT 3. The levels of SREBPTC and CRY1 mRNAs were determined by qRT-PCR and normalized to TBP mRNA levels. Data are represented as mean \pm s.d., $N=3-4$ for each group. ${ }^{\star} P<0.05,{ }^{\star \star} P<0.01$ (Student's $t$-test). See Supplementary Fig. 13 for original full immunoblot.

SREBP $1 c^{-/-}$mice. While SREBP1c $c^{-/-}$mice showed higher blood glucose level than SREBP1c ${ }^{+/+}$mice during the pyruvate tolerance test, SREBP $1 c^{-/-}$mice with CRY1 overexpression decreased blood glucose level (Fig. 31,m, Supplementary Fig. 6A,B). These data strongly indicate that the SREBP1c-CRY1 signalling pathway could inhibit hepatic gluconeogenesis in vivo.

CRY1 regulates FOXO1 protein levels. To decipher the underlying mechanism(s) by which insulin-induced CRY1 could repress hepatic gluconeogenesis, we focused on FOXO1, as its regulatory effects on insulin signalling and gluconeogenesis are well established. In mouse primary hepatocytes, the level of FOXO1 protein was decreased by CRY1 overexpression (Fig. 4a) while FOXO1 mRNA levels were not altered (Fig. 4b). These data indicated that CRY1 might modulate the level of FOXO1 protein, probably, independent of FOXO1 mRNA. Furthermore, the level of FOXO1 protein was enhanced in CRY1 suppressed hepatocytes
(Fig. 4c,d). In accordance with these in vitro data, the level of FOXO1 protein was higher in the liver of $C R Y 1^{-/-}$mice than in the liver of $C R Y 1^{+1+}$ mice, whereas the levels of FOXO1 mRNA were not different between $C R Y 1^{+/+}$and $C R Y 1^{-/-}$mice (Fig. 4e,f). Compared with SREBP1c ${ }^{+/+}$mice, the levels of FOXO1 protein and G6Pase and PEPCK mRNAs were augmented in the liver of $S R E B P 1 c^{-/-}$mice whereas that of CRY1 protein was reduced (Fig. 4g, Supplementary Fig. 7). To verify that CRY1 could inhibit hepatic gluconeogenesis via FOXO1, the effects of CRY1 and/or FOXO1 suppression on gluconeogenic gene expression were examined. Increased expression of G6Pase and PEPCK genes by CRY1 suppression was abolished when FOXO1 gene was downregulated by siRNA (Fig. 4h), implying that FOXO1 might be a downstream factor of CRY1 to regulate gluconeogenesis. These in vivo and in vitro data imply that CRY1 would alleviate hepatic gluconeogenesis through FOXO1. 
a

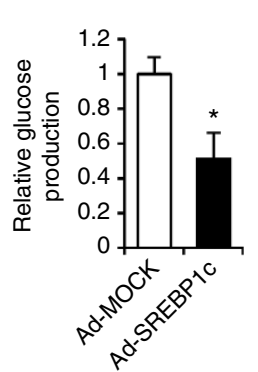

b

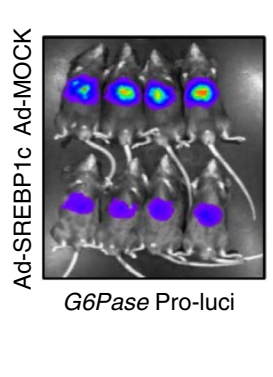

$\left(10^{8}\right)$

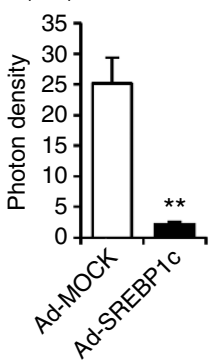

e

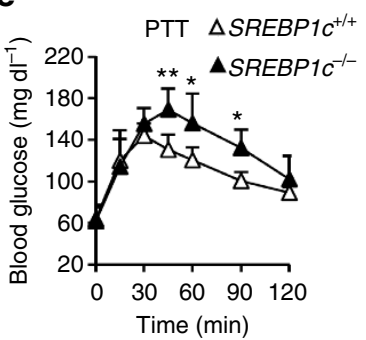

f

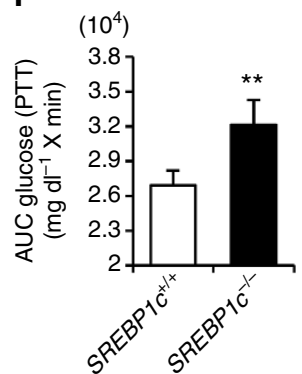

C

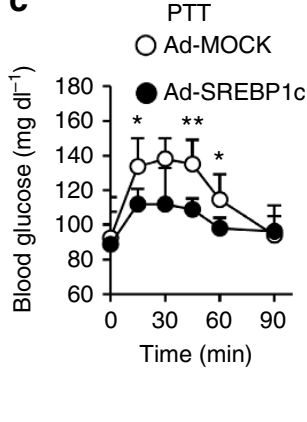

d

$\left(10^{4}\right)$

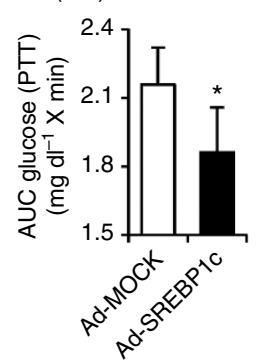

g

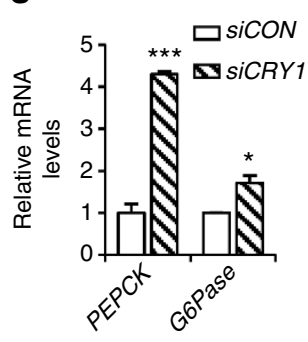

h

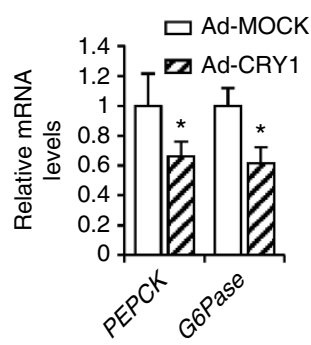

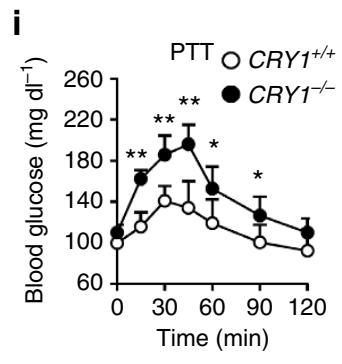

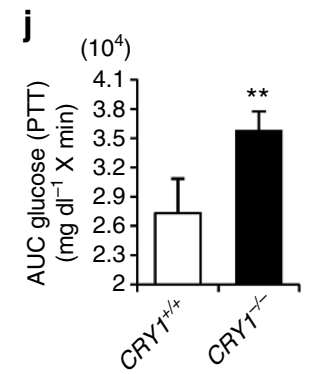

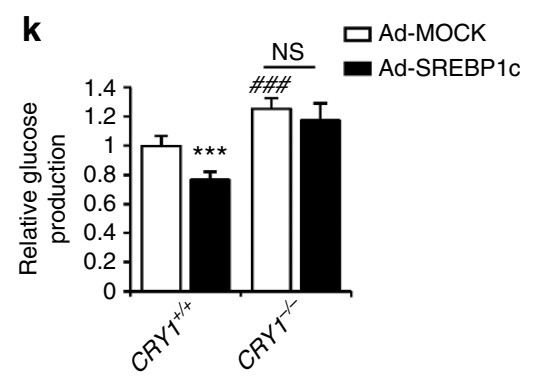

I
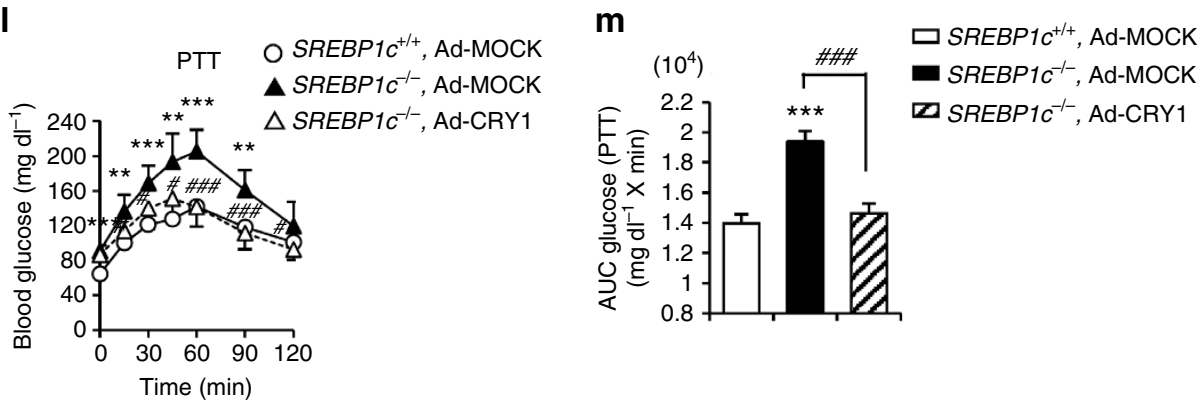

Figure 3 | The SREBP1C-CRY1 signalling pathway regulates hepatic glucose production. (a) Mouse primary hepatocytes were infected with Ad-MOCK or Ad-SREBP1c. Relative glucose production was measured using a glucose oxidase (GO) kit as described in Methods. Data represent mean $\pm \mathrm{s}$.d., $N=3$ for each group. ${ }^{\star} P<0.05$ (Student's t-test). (b) C57BL/6 mice were infected with Ad-G6Pase-luc and either Ad-MOCK or Ad-SREBP1c. G6Pase promoter activity was measured by optical in vivo imaging and photon density. (c,d) C57BL/6 mice were infected with Ad-MOCK or Ad-SREBP1c and performed pyruvate tolerance test (c). All mice were fasted at ZT 10 and performed PTT at ZT 3. Results were converted to area-under-the curve (AUC) (d). Data represent mean \pm s.d., $N=5$ for each group. ${ }^{\star} P<0.05,{ }^{\star \star} P<0.01$ (Student's $t$-test). (e,f) Pyruvate tolerance test (e) was performed in $S R E B P 1 c^{-/}-$ and SREBP1C $+/+$ mice. All mice were fasted at ZT 10 and performed PTT at ZT 3. Results were converted to AUC (f). Data represent mean \pm s.d., $N=5$ for each group. ${ }^{\star} P<0.05,{ }^{\star} P<0.01$ (Student's $t$-test). (g) H4IIE cells were transfected with siCON or siCRY1. Relative mRNA levels were determined by qRT-PCR and normalized to cyclophilin mRNA levels. Data represent mean \pm s.d., $N=3$ for each group. ${ }^{\star} P<0.05$, ${ }^{\star \star \star} P<0.001$ (Student's $t$-test). (h) Mouse primary hepatocytes were infected with Ad-MOCK or Ad-CRY1. Relative mRNA levels were determined by qRT-PCR and normalized to TBP mRNA levels. Data represent mean \pm s.d., $N=3$ for each group. ${ }^{\star} P<0.05$ (Student's $t$-test). (i,j) Pyruvate tolerance test (i) was performed in $C R Y 1^{-/}-$and $C R Y 1^{+} /+$ mice. All mice were fasted at ZT 10 and performed PTT at ZT 3. Results were converted to AUC (j). Data represent mean \pm s.d., $N=7$ for each group. ${ }^{\star} P<0.05,{ }^{\star \star} P<0.01$ (Student's $t$-test). (k) Mouse primary hepatocytes isolated from $C R Y 1^{+/+}$and $C R Y 1^{-/}-$mice were infected with Ad-MOCK or Ad-SREBP1c. Relative glucose production was measured using a glucose oxidase (GO) kit. Data represent mean \pm s.d., $N=8$ for each group. ${ }^{\star \star \star} P<0.001$ versus Ad-MOCK, \#\#\#P<0.001 versus CRY1 ${ }^{+/+}$(Student's $t$-test). (I, m) Pyruvate tolerance test (I) in SREBP1c ${ }^{+/+}$mice injected with Ad-MOCK and in SREBP1c ${ }^{-/-}$mice injected with either Ad-MOCK or Ad-CRY1. Results were converted to AUC (m). All mice were fasted at ZT 10 and performed PTT at ZT 3. Data represent mean \pm s.d., $N=7-10$ for each group. ${ }^{\star \star} P<0.01,{ }^{\star \star \star} P<0.001$ versus SREBP1C ${ }^{+/+}$, Ad-MOCK, ${ }^{\#} P<0.05,{ }^{\# \# \#} P<0.001$ versus SREBPIC ${ }^{-/-}$, Ad-MOCK (Student's t-test). 
a
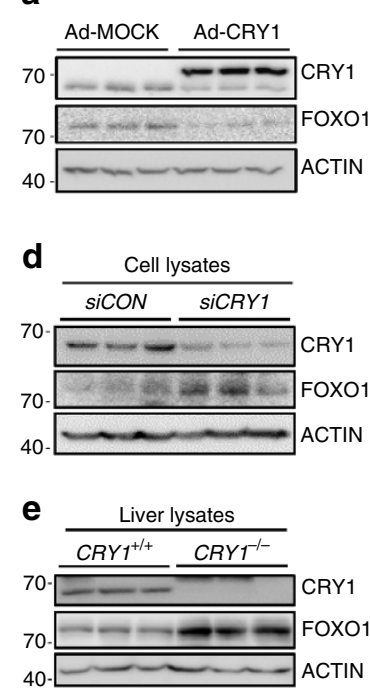

b
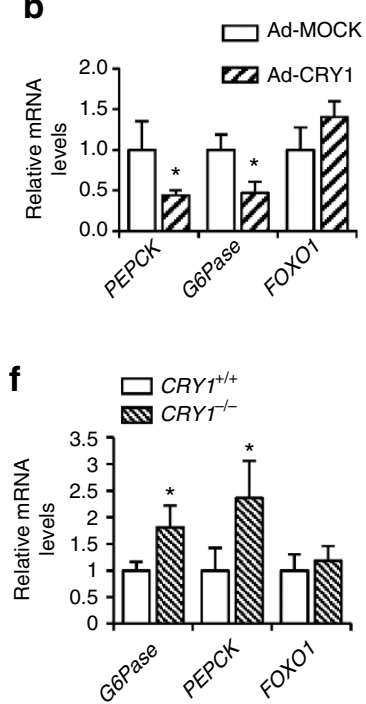

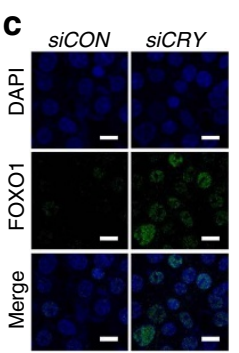

g

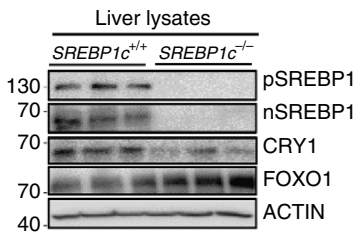

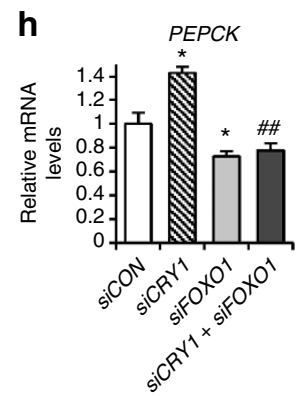
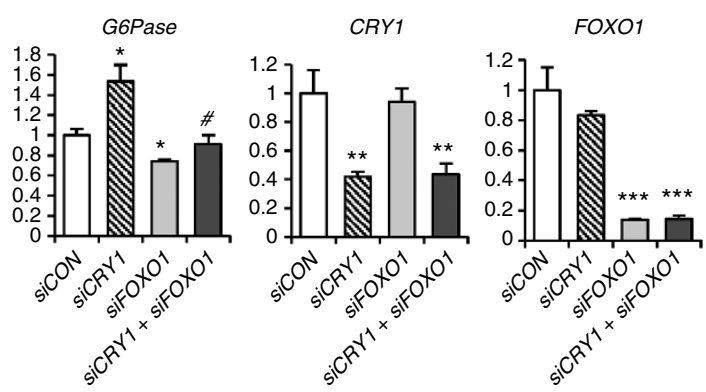

Figure 4 | CRY1 regulates FOX01 protein level. (a,b) Mouse primary hepatocytes were adenovirally infected with Ad-MOCK or Ad-CRY1. The expression profiles of FOXO1 were analysed at the protein level (a) using western blotting and at the mRNA level (b) using qRT-PCR. Data are represented as mean \pm s.d., $N=4$ for each group. ${ }^{\star} P<0.05$ (Student's $t$-test). (c,d) H4IIE cells were transfected with siCON or siCRY1. Immunocytochemical analysis (c) of endogenous FOXO1. DAPI, 4', 6-diamidino-2-phenylindole. Scale bars, $10 \mu \mathrm{m}$. Endogenous FOXO1 and CRY1 protein levels were analysed using western blotting (d). (e,f) The expression patterns of FOXO1 protein in the liver of $C R Y 1^{+/+}$and $C R Y 1^{-/-}$mice were analysed by western blotting (e) and qRT-PCR (f). Relative mRNA levels were determined using qRT-PCR and normalized to the levels of the TBP mRNA. Data are represented as mean \pm s.d., $N=3$ for each group. ${ }^{\star} P<0.05$, (Student's $t$-test). (g) Expression of CRY1 and FOXO1 proteins in the liver of SREBP1c ${ }^{+/+}$and SREBP1c ${ }^{-/-}$mice was analysed by western blotting. (h) H4IIE cells were co-transfected with siCRY1 and/or siFOXO1. Relative mRNA levels were determined using qRT-PCR and normalized to the cyclophilin mRNA level. Data are represented as mean \pm s.d., $N=3$ for each group. ${ }^{\#} P<0.05,{ }^{\# \#} P<0.01$ versus siCRY1, ${ }^{\star} P<0.05$, ${ }^{\star \star} P<0.01,{ }^{\star \star} P<0.001$ versus siCON (Student's $t$-test). See Supplementary Fig. 13 for original full immunoblot.

FOXO1 protein is decreased by insulin-activated CRY1. FOXO1 translocation from the nucleus to the cytoplasm by AKT is a well-known mechanism by which insulin acutely inhibits hepatic glucose production ${ }^{43}$. As insulin upregulates CRY1 that, in turn, downregulates the FOXO1 protein (Figs 1c and 4a), we investigated the time course of these events by examining the expression profiles of FOXO1 and CRY1 proteins in insulin-treated primary hepatocytes. As shown in Fig. 5a, phosphorylation of AKT and FOXO1 was clearly induced in cells treated with insulin for a relatively short periods $(0-4 \mathrm{~h})$. Consistent with previous report ${ }^{44}$, the level of FOXO1 protein was decreased by short-term insulin treatment $(0 \sim 4 \mathrm{~h})$, which would be mediated by FOXO1 ubiquitination and degradation in cytosol. Furthermore, the phosphorylation levels of AKT and FOXO1 were gradually and substantially decreased by a longterm incubation $(8-12 \mathrm{~h})$ with insulin. It appears that FOXO1 translocation from the nucleus to the cytoplasm by AKT might be more pronounced after a short exposure $(0-4 \mathrm{~h})$ to insulin rather than following a long-term insulin treatment $(8-12 \mathrm{~h})$. Intriguingly, in hepatocytes treated with insulin for long periods $(8-12 \mathrm{~h})$, the level of the CRY1 protein was markedly increased, while that of the total FOXO1 protein was continuously decreased, implying that the amount of the CRY1 protein appears to be inversely related to the total quantity of the FOXO1 protein. Accordingly, the expression of PEPCK and G6Pase genes was downregulated after either a long-term $(8-12 \mathrm{~h})$ or a shortterm $(0-4 \mathrm{~h})$ insulin treatment (Fig. 5b). These data suggest that sustainable reduction of the FOXO1 protein might be involved in the suppression of hepatic gluconeogenesis for long-term insulin action.

Next, we explored whether CRY1 could indeed modulate the FOXO1 protein in insulin-treated hepatocytes. To address this, we have tested $C R Y 1^{+/+}$and $C R Y 1^{-/-}$primary hepatocytes. As shown in Fig. 5c, the level of FOXO1 protein was decreased in insulin-treated $C R Y 1^{+/+}$primary hepatocytes while that of FOXO1 protein was not continuously suppressed in insulintreated $C R Y 1^{-/-}$primary hepatocytes. In the late phase of insulin action, decreased levels of PEPCK and G6Pase mRNA were slightly but substantially increased in $C R Y 1^{-/-}$primary hepatocytes (Fig. 5d). Moreover, insulin failed to suppress glucose production in long-term $(8-12 \mathrm{~h})$ insulin-treated $C R Y 1^{-/-}$primary hepatocytes. (Fig. 5e). Altogether, these data indicate that CRY1 could repress the expression of hepatic gluconeogenic genes with reduced FOXO1 protein for the long-term insulin action (8-12 h). 
a

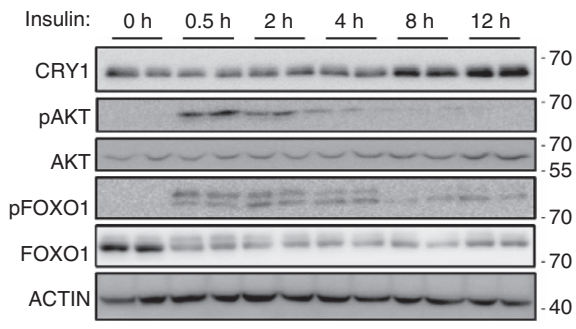

b
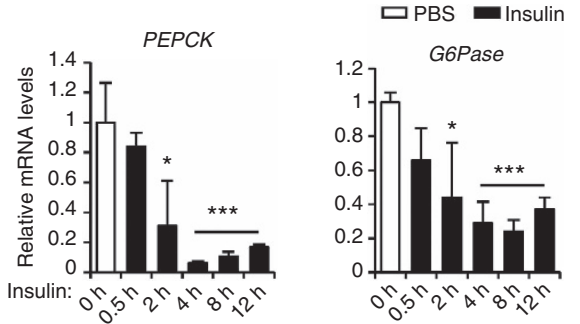

C

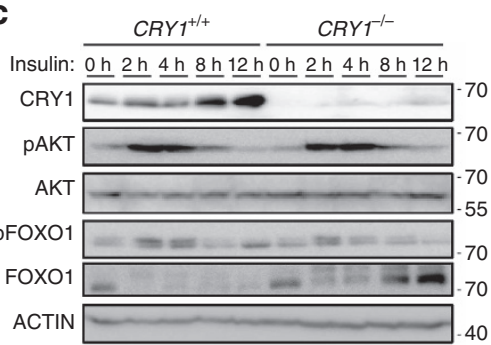

d

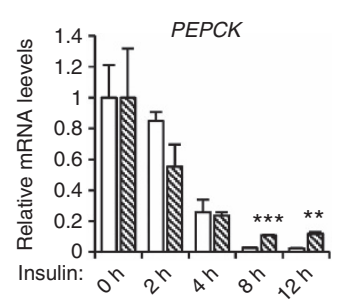

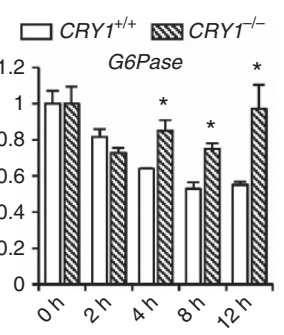

e

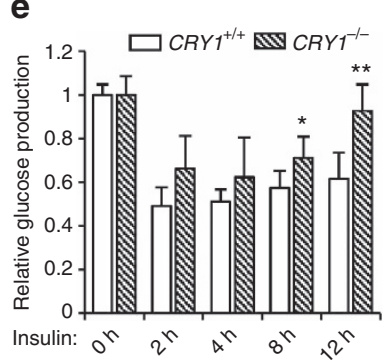

f

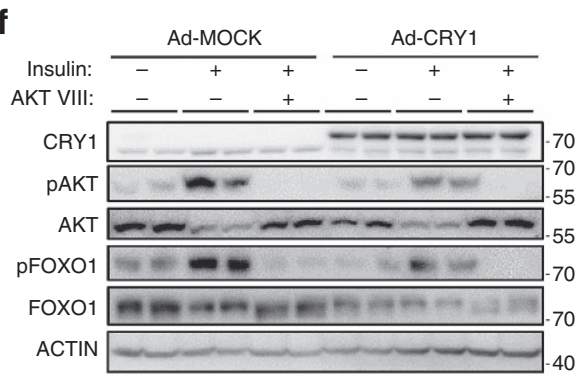

g

$$
\begin{aligned}
& \text { Ad-MOCK + vehicle } \\
& \text { Ad-MOCK + MK2206 }
\end{aligned}
$$
$\triangle \mathrm{Ad}-\mathrm{CRY} 1+\mathrm{MK} 2206$

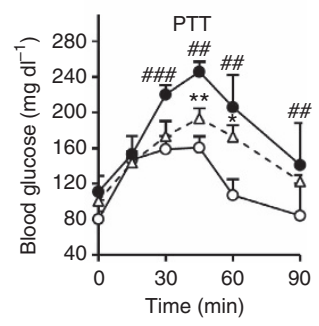

h

$$
\begin{aligned}
& \text { 口 Ad-MOCK + vehicle } \\
& \text { Ad-MOCK + MK2206 } \\
& \text { m Ad-CRY1 + MK2206 }
\end{aligned}
$$

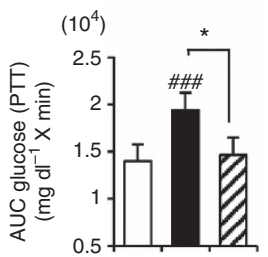

i

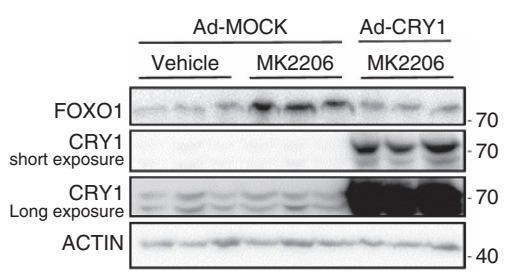

Figure 5 | Long-term insulin treatment stimulates CRY1 expression and inhibits hepatic gluconeogenesis. (a,b) Mouse primary hepatocytes were treated with $10 \mathrm{nM}$ insulin for different periods. Protein levels (a) were determined with western blotting. The mRNA levels (b) were analysed by qRT-PCR. Data are represented as mean \pm s.d., $N=3$ for each group. ${ }^{\star} P<0.05,{ }^{\star \star \star} P<0.001$ (Student's $t$-test). (c-e) Mouse primary hepatocytes isolated from $\mathrm{CRY}^{+} /+$and $\mathrm{CRY} 1^{-/-}$mice were treated with $10 \mathrm{nM}$ insulin for different periods. Protein levels (c) were analysed by western blotting, and relative mRNA levels (d) were determined by qRT-PCR and normalized to the TBP mRNA level. Data are represented as mean \pm s.d., $N=3$ for each group. ${ }^{\star} P<0.05,{ }^{* \star} P<0.01,{ }^{* \star *} P<0.001$ versus $C R Y 1^{+/+}$control (Student's $t$-test). Relative glucose production (e) was measured using a glucose oxidase (GO) kit. Data are represented as mean \pm s.d., $N=5$ for each group. ${ }^{\star} P<0.05,{ }^{\star \star} P<0.01$, versus $C R Y 1^{+} /+$control (Student's $t$-test). (f) Mouse primary hepatocytes were infected with Ad-MOCK and Ad-CRY1, and then treated with insulin (10 nM) or insulin (10 nM) and AKTVIII (5 $\mu$ M) for $12 \mathrm{~h}$. Protein levels were determined with western blotting. (g-i) C57BL/6 mice were infected with Ad-MOCK or Ad-CRY1 and subjected to the pyruvate tolerance test ( $\mathbf{g}$ ) with or without the AKT inhibitor MK2206. MK2206 (30 mg kg ${ }^{-1}$ ) was given by oral gavage 10 min before the pyruvate tolerance test. All mice were fasted at ZT 10 and performed PTT at ZT 3. Results were converted to AUC values (h). After the pyruvate tolerance test, hepatic protein levels (i) were analysed by western blotting. Data are represented as mean \pm s.d., $N=5-7$ for each group. ${ }^{\#} P<0.01$, \#\#\# $P<0.001$ versus Ad-MOCK + vehicle control, ${ }^{\star} P<0.05,{ }^{\star \star} P<0.01$, versus Ad-MOCK + MK2206 control (Student's $t$-test). See Supplementary Fig. 13 for original full immunoblot.

To test whether enhanced CRY1 could suppress hepatic gluconeogenesis even in the absence of a short-term insulin action $(0 \sim 4 \mathrm{~h})$, we employed AKT inhibitors. In primary hepatocytes, insulin increased phosphorylation levels of both
AKT and FOXO1, while a co-treatment with the AKT inhibitor AKTVIII blocked phosphorylation of both proteins, as expected (Fig. 5f). However, in CRY1-overexpressing hepatocytes, the total FOXO1 level was decreased in insulin and/or the AKT inhibitor 
treated cells, implying that CRY1 could downregulate FOXO1 protein independent of FOXO1 phosphorylation (Fig. 5f). To confirm this observation in vivo, we tested another AKT inhibitor, MK2206, in mice. As shown in Fig. 5g,h, administration of MK2206 significantly increased blood glucose level on pyruvate challenge; however, adenoviral CRY1 overexpression in mice significantly attenuated blood glucose level even in the presence of MK2206. It is noteworthy that the level of FOXO1 protein was greatly augmented by MK2206, whereas CRY1 elevation suppressed FOXO1 protein expression in vivo (Fig. 5i). Taken together, these data clearly indicate that CRY1-dependent FOXO1 reduction may contribute to the suppression of hepatic gluconeogenesis independent of AKT activation.

CRY1 stimulates proteasomal degradation of FOXO1. Since CRY1 overexpression decreased the level of the FOXO1 protein, but not the FOXO1 mRNA, we investigated whether the downregulation of the FOXO1 protein might proceed via proteasomal degradation. As shown in Fig. 6a, the reduction of the FOXO1 protein by CRY1 overexpression was alleviated by MG132 treatment, indicating that the regulation of FOXO1 protein by CRY1 may be, at least in part, dependent on proteasomal degradation. When we tested physical interaction between FOXO1 and CRY1 proteins, co-immunoprecipitation assays revealed that CRY1 could associate with FOXO1 protein (Fig. 6b). Then, we examined whether CRY1 might induce FOXO1 degradation via the ubiquitination-proteasome pathway. As shown in Fig. 6c, CRY1 overexpression dramatically promoted FOXO1 poly-ubiquitination, implying that CRY1 could potentiate FOXO1 degradation, probably, through proteinprotein interactions.

To further explore the subcellular location of FOXO1 degradation induced by CRY1, the levels of the nuclear and cytosolic FOXO1 protein were investigated. As shown in Fig. 6d, the nuclear fraction of the FOXO1 protein was decreased by CRY1 overexpression whereas incubation with MG132 blocked this decrease. At the same time, the levels of cytosolic FOXO1 were not altered on CRY1 overexpression, irrespective of the presence of MG132. Consistent with these results, polyubiquitination of the nuclear form of the FOXO1 mutant protein (nFOXO1-MYC) was greatly augmented by CRY1 (Fig. 6e). Therefore, it is plausible that the degradation of the FOXO1 protein via poly-ubiquitination is stimulated by CRY1 in the nucleus.

CRY1 is involved in MDM2-mediated FOXO1 degradation. Among several ubiquitin E3 ligases of the FOXO1 protein ${ }^{45,46}$, we found that the MDM2 ubiquitin E3 ligase appeared to be involved in the CRY1-mediated FOXO1 degradation. As shown in Fig. 7a, MDM2 suppression markedly rescued the level of the FOXO1 protein in CRY1-overexpressing cells, implying that MDM2 may participate in the CRY1-dependent FOXO1 reduction. To study the role of CRY1 in MDM2-mediated FOXO1 degradation, we tested whether CRY1 might regulate the subcellular localization of MDM2. Wild-type CRY1 and cytosolic CRY1 (DNLS-CRY1) did not change the subcellular location of the nuclear MDM2 (Supplementary Fig. 8). Instead, we revealed that CRY1 potentiates the association between FOXO1 and MDM2 (Fig. 7b).
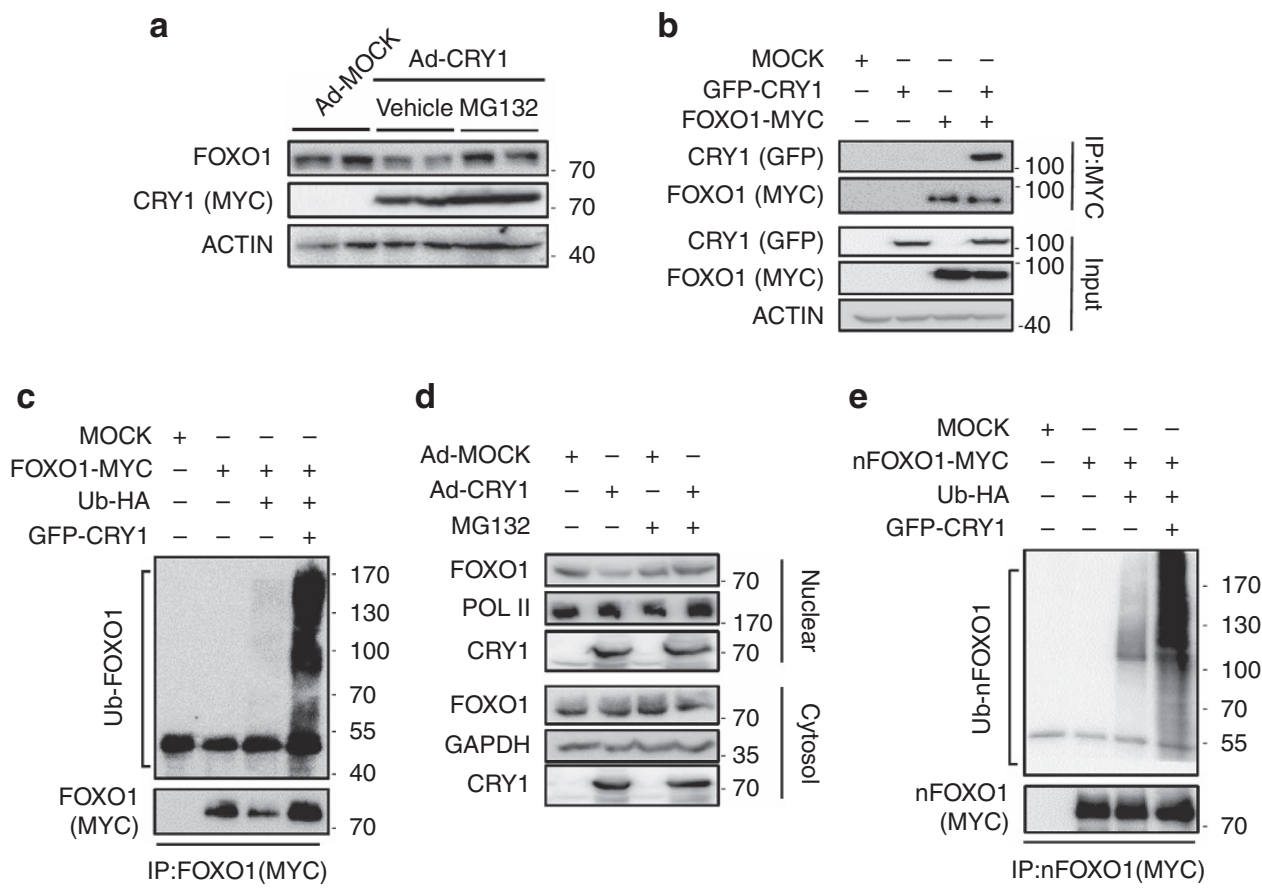

Figure 6 | CRY1 accelerates ubiquitin-mediated FOXO1 degradation. (a) Mouse primary hepatocytes were adenovirally infected with Ad-MOCK or Ad-CRY1. The cells were treated with $20 \mu \mathrm{M}$ MG132 or vehicle for $4 \mathrm{~h}$. Total cell lysates were analysed by western blotting with indicated antibodies. (b) HEK293T cells were transfected with GFP-CRY1 and/or FOXO1-MYC expression vectors. Co-immunoprecipitation with an anti-MYC antibody and western blotting were performed with the indicated antibodies. IP, immunoprecipitation. (c) COS-1 cells were co-transfected with plasmids encoding FOXO1-MYC, GFP-CRY1, and Ubiquitin-HA. After transfection, the cells were treated with MG132 (20 $\mu \mathrm{M})$ for $6 \mathrm{~h}$ and then the cell lysates were subjected to immunoprecipitation with an anti-MYC antibody followed by western blotting with indicated antibodies. IP, immunoprecipitation. (d) Mouse primary hepatocytes were infected with Ad-MOCK or Ad-CRY1. After infection, the cells were treated with MG132 (20 $\mu \mathrm{M})$ for $4 \mathrm{~h}$. Nuclear and cytosolic fractions

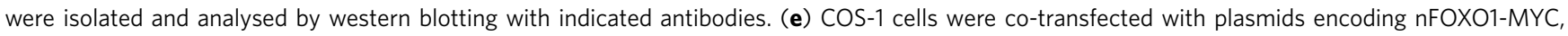
GFP-CRY1, and Ubiquitin-HA. After transfection, the cells were challenged with MG132 $(20 \mu \mathrm{M})$ for $6 \mathrm{~h}$. The cell lysates were subjected to immunoprecipitation with an anti-MYC antibody. IP, immunoprecipitation. See Supplementary Fig. 13 for original full immunoblot. 
a

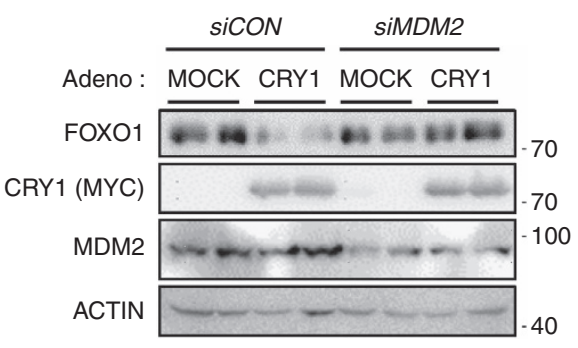

C

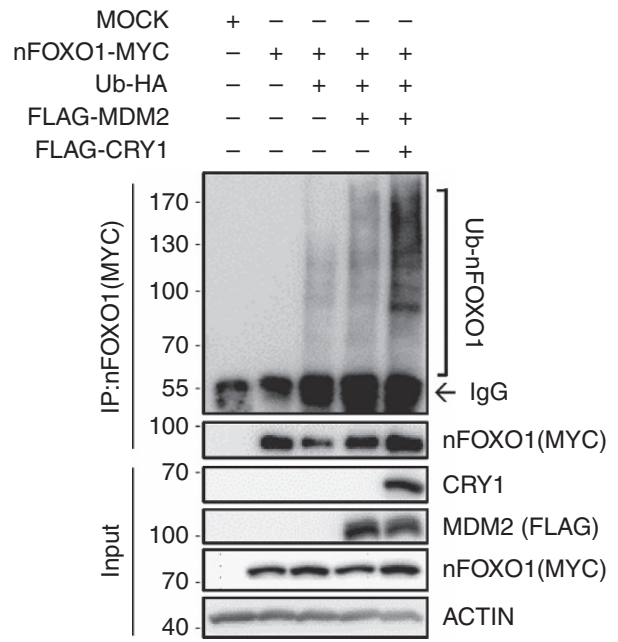

b

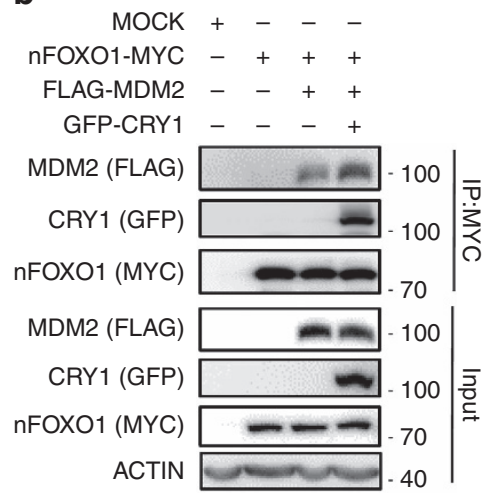

d

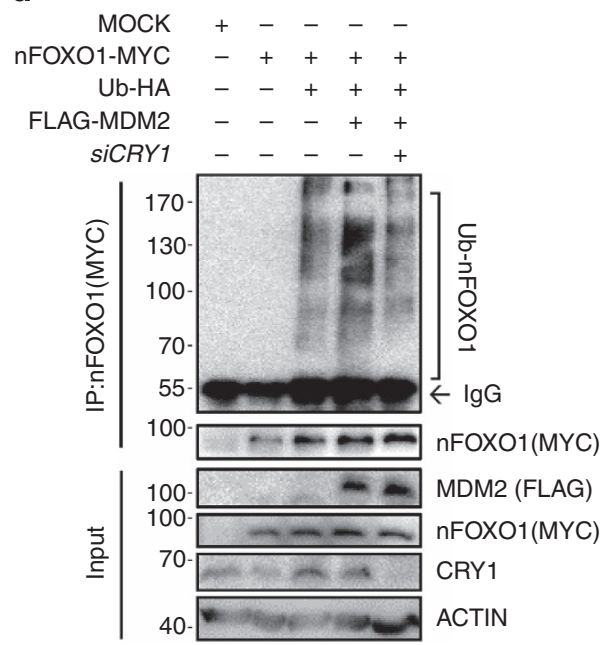

Figure 7 | CRY1 is involved in MDM2-mediated FOXO1 ubiquitination. (a) Mouse primary hepatocytes were infected with Ad-MOCK or Ad-CRY1 and/or siCON or siMDM2. Total cell lysates were analysed by western blotting with indicated antibodies. (b) HEK293T cells were transfected with FLAG-MDM2, nFOXO1-MYC, and GFP-CRY1 expression vectors. Total cell lysates were subjected to co-immunoprecipitation with an anti-MYC antibody followed by

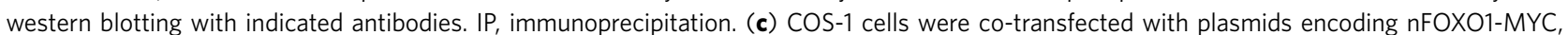
FLAG-MDM2, FLAG-CRY1, and Ubiquitin-HA. After transfection, the cells were challenged with MG132 (20 $\mu \mathrm{M})$ for $6 \mathrm{~h}$. Cell lysates were subjected to immunoprecipitation with an anti-MYC antibody. IP, immunoprecipitation (d) COS-1 cells were co-transfected with plasmids encoding nFOXO1-MYC, FLAG-MDM2, Ubiquitin-HA, and siCRY1. Cells were treated with MG132 $(20 \mu \mathrm{M})$ for $6 \mathrm{~h}$. Cell lysates were subjected to immunoprecipitation with an anti-MYC antibody. IP, immunoprecipitation. See Supplementary Fig. 13 for original full immunoblot.

In another experiment, we explored whether CRY1 could modulate MDM2-mediated FOXO1 degradation. As shown in Fig. 7c,d, CRY1 overexpression promoted MDM2-mediated poly-ubiquitination of the nuclear form of FOXO1 protein (Fig. 7c), whereas CRY1 suppression attenuated FOXO1 polyubiquitination by MDM2 (Fig. 7d). These data indicate that CRY1 would participate in MDM2-induced FOXO1 degradation and repress FOXO1-mediated hepatic glucose production.

CRY1 mitigates hyperglycaemia in diabetic mouse models. Streptozotocin (STZ)-treated insulin-deficient mice elevated blood glucose as well as gluconeogenic programs including FOXO1 protein levels, accompanied with increased expression of PEPCK and G6Pase genes (Fig. 8a-c). Since the SREBP1c-CRY1 axis was upregulated by insulin (Figs 1c,2e), we overexpressed CRY1 in the liver of STZ-treated insulin-deficient mice. As shown in Fig. 8d, CRY1 overexpression diminished blood glucose levels in STZ-treated mice. In addition, CRY1 reduced not only the expression of PEPCK and G6Pase genes but also the level of FOXO1 protein (Fig. 8e,f). These data suggest that CRY1 would be one of important factors to suppress hepatic glucose production on insulin.
Intriguingly, in the liver of obese animals such as $d b / d b$ and diet-induced obesity mice, SREBP1c level is elevated while hepatic gluconeogenesis is not repressed ${ }^{4-50}$. To explore which process(es) might be dysregulated in the regulation of hepatic gluconeogenesis, we have examined several mRNA and protein levels for SREBP1c-CRY1 axis and gluconeogenic genes in diabetic animals. Similar to previous reports ${ }^{47-50}$, the mRNA levels of SREBP1c and gluconeogenic genes were elevated in $d b / d b$ mice (Fig. 8g). However, hepatic CRY1 protein was substantially decreased and the level of MDM2 protein was not significantly changed in $d b / d b$ mice (Fig. 8h). Similarly, diet-induced obesity mice exhibited elevated expression of SREBP1c and gluconeogenic genes whereas CRY1 was not activated (Supplementary Fig. 9A,B). To test the idea that dysregulated CRY1 protein might mediate hyperglycaemia with enhanced FOXO1 protein in diabetic animals, CRY1 was adenovirally overexpressed in the liver of $d b / d b$ mice. As shown in Fig. 8i, the levels of pyruvate-induced blood glucose were decreased in CRY1-overexpressing $d b / d b$ mice. Moreover, the levels of feeding blood glucose were diminished by CRY1 overexpression (Fig. 8j). In addition, ectopic CRY1 expression reduced the levels of FOXO1 protein as well as gluconeogenic gene expression in $d b / d b$ 
a

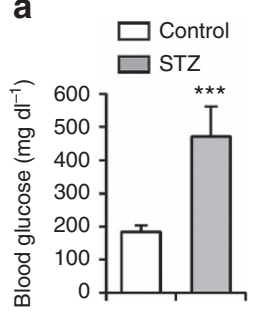

d

$\square$ STZ, Ad-MOCK Z STZ, Ad-CRY1

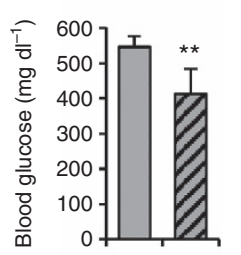

g
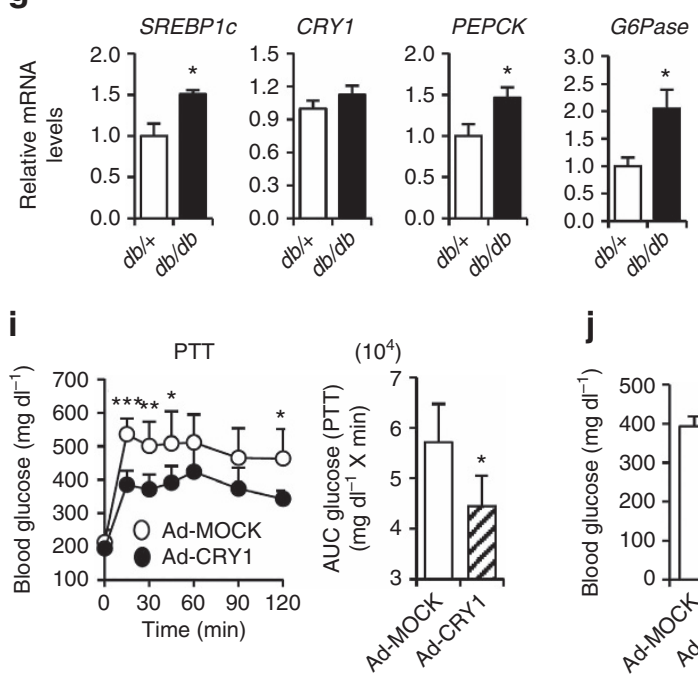

I
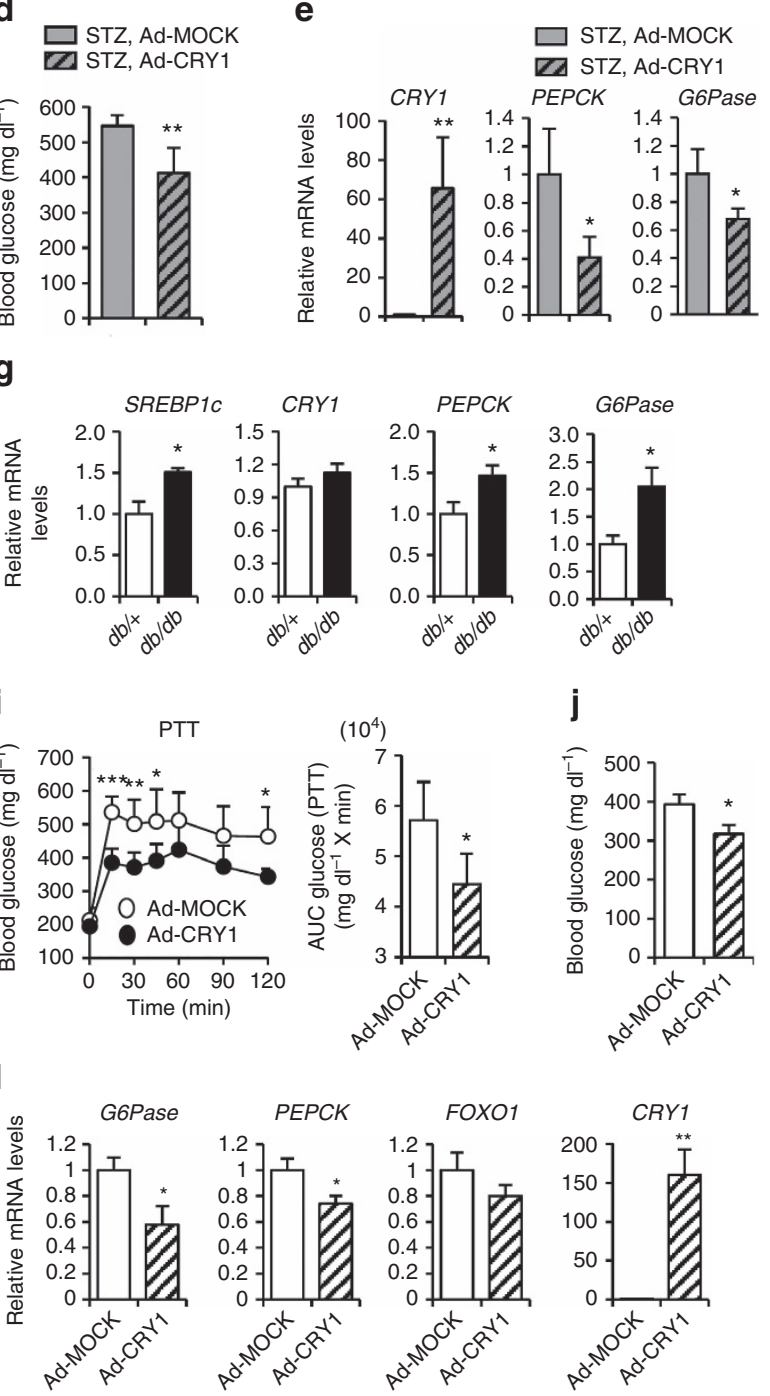

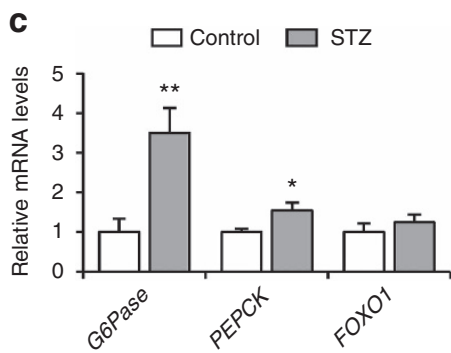

f

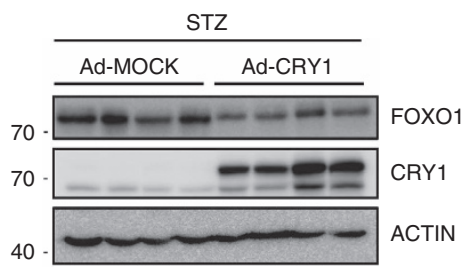

h

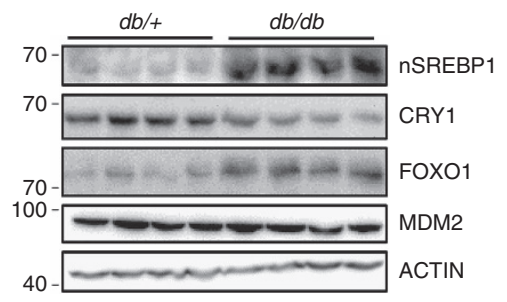

$\mathbf{k}$

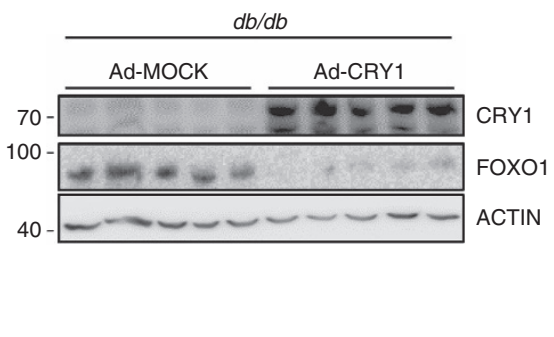

Figure 8 | CRY1 alleviates gluconeogenesis in diabetic mouse models. (a-c) Eight-week-old male mice were injected STZ (150 mg kg $\left.{ }^{-1}\right)$ and sacrificed in ZT 3 after 1 week later. Blood glucose levels (a) were measured in ad libitum at ZT 3. After all of the mice were sacrificed at ZT 3, hepatic protein levels (b) were analysed by western blotting. The mRNA levels (c) were determined by qRT-PCR analyses and normalized to the TBP mRNA level. Data represent mean \pm s.d., $N=3$ for each group. ${ }^{\star} P<0.05,{ }^{\star \star} P<0.01$, ${ }^{\star \star \star} P<0.001$ (Student's $t$-test). (d-f) STZ-injected mice were infected with adenovirus encoding MOCK or CRY1 (adenoviral dose of $2 \times 10^{9}$ viral particles per mouse). The blood glucose levels (d) were measured in ad libitum at ZT 3. After all of the mice were sacrificed at ZT 3, the mRNA levels (e) were determined by qRT-PCR analyses and normalized to the TBP mRNA level. Hepatic protein levels (f) were analysed by western blotting. Data represent mean \pm s.d., $N=4$ for each group. ${ }^{\star} P<0.05,{ }^{\star \star} P<0.01$ (Student's $t$-test). (g,h) Ten-week-old male $d b /+$ and $d b / d b$ mice were sacrificed in ad libitum at ZT 3. The relative mRNA levels of various hepatic genes ( $\mathbf{g}$ ) were determined by qRT-PCR analyses and normalized to the TBP mRNA level. Data represent mean \pm s.d., $N=4$ for each group. ${ }^{\star} P<0.05$ (Student's $t$-test). Protein levels (h) were determined with western blotting. (i-I) Ten-week-old male $d b / d b$ mice were infected through the tail vein with adenovirus encoding MOCK or CRY1 (adenoviral dose of $2 \times 10^{10}$ viral particles per mouse) and subjected to the pyruvate tolerance test (i). All mice were fasted at ZT 10 and performed PTT at ZT 3. Results were converted to AUC. The blood glucose levels (j) were measured in ad libitum at ZT 3. After all of the mice were sacrificed at ZT 3, hepatic protein levels (k) were analysed by western blotting. The mRNA levels (I) were determined by qRT-PCR analyses and normalized to the TBP mRNA level. Data represent mean \pm s.d., $N=5$ for each group. ${ }^{\star} P<0.05,{ }^{\star \star} P<0.01,{ }^{\star \star \star} P<0.001$ (Student's $t$-test). See Supplementary Fig. 13 for original full immunoblot. 
mice (Fig. 8k,l). These results indicate that CRY1 could ameliorate hyperglycaemia by repressing the level of FOXO1 protein in $d b / d b$ mice.

\section{Discussion}

As a major anabolic hormone, insulin stimulates lipogenesis and represses gluconeogenesis in the liver. Following insulin exposure, lipogenesis is upregulated by SREBP1c, and the expression of SREBP1c target genes such as FASN, SCD and ELOVL6 is induced ${ }^{20,51,52}$. In contrast, insulin blocks hepatic gluconeogenesis through AKT-mediated phosphorylation of FOXO1 and PGC1 $\alpha^{53,54}$, both are major regulators of gluconeogenic genes including PEPCK and G6Pase. Here we propose that the SREBP1c-CRY1 signalling pathway plays an important role to inhibit hepatic gluconeogenesis under anabolic state. Our data from hepatic gluconeogenic gene expression, in vitro glucose output assays, time kinetics of insulin signalling cascades, and pyruvate tolerance test, which reflects both hepatic glucose output and peripheral glucose disposal, have consistently suggested the idea that maintenance of SREBP1c-induced CRY1 is crucial to prevent unnecessary hepatic gluconeogenesis during insulin action. In this regard, it has been reported that single-nucleotide polymorphisms of SREBP1c and CRY1 genes are associated with hyperglycaemia in human ${ }^{55,56}$.

Similar to previous reports ${ }^{47,48,50}$, the expression of SREBP1c and gluconeogenic genes was increased in the liver of diabetic $d b / d b$ mice (Fig. 8g,h). Unexpectedly, the level of hepatic CRY1 protein was downregulated in $d b / d b$ mice. Moreover, in the liver of $d b / d b$ mice, diurnal expression of CRY1 protein level was overall reduced whereas FOXO1 protein levels were elevated compared to $d b /+$ mice (Supplementary Fig. 10A,B). In this work, we have demonstrated that ectopic overexpression of CRY1 in $d b / d b$ mice alleviated hepatic gluconeogenesis by reducing FOXO1 protein (Fig. 8i-1). Also, we have shown that hepatic CRY1 could attenuate the level of blood glucose by decreasing FOXO1 protein, independent of AKT activity (Fig. 5f-i). Although it remains to be elucidated how elevated SREBP1c fails to increase CRY1 in the liver of $d b / d b$ mice, it is very likely that increased FOXO1 protein results from reduced hepatic CRY1 protein in $d b / d b$ mice.

It has been reported that CRY1 seems to suppress hepatic glucose production by interfering with glucocorticoid receptor signalling and the glucagon signalling pathway ${ }^{33-35}$. CRY1 interacts with glucocorticoid receptor ${ }^{34}$ and the $\alpha$-subunit of the glucagon receptor ${ }^{33}$, which are involved in the regulation of gluconeogenesis. Nonetheless, specific roles of CRY1 during the nutrient-rich state after exposure to insulin have not been clearly elucidated. To test whether CRY1 might repress gluconeogenesis by inhibiting glucagon signalling and/or glucocorticoid receptordependent pathway, CRY1-overexpressing primary hepatocytes were treated with forskolin, db-cAMP or dexamethasone to mimic the conditions for glucagon or glucocorticoid stimulation. In primary hepatocytes, CRY1 partially repressed gluconeogenic gene expression in the presence of forskolin, db-cAMP or dexamethasone (Supplementary Fig. 11A-C), implying that in addition to the glucagon and glucocorticoid signalling pathways, there may be another signalling cascade regulated by CRY1, which could suppress hepatic glucose production. Thus, CRY1 appears to be involved in multiple regulatory pathways that control hepatic gluconeogenesis in response to various hormones.

Activation of FOXO1-mediated gluconeogenesis is inhibited by insulin. AKT, a key downstream molecule of the insulin-activated signalling, phosphorylates FOXO1, which then is translocated from the nucleus to the cytoplasm through its association with the 14-3-3 protein ${ }^{57}$. In primary hepatocytes, FOXO1 phosphorylation was rapidly increased by insulin. However, hepatic gluconeogenic programing is persistently and efficiently suppressed regardless of the decreased FOXO1 phosphorylation at the late stage of insulin action. Intriguingly, hepatic CRY1 expression was enhanced at relatively late periods of insulin action (Fig. 5a). Furthermore, in primary hepatocytes, a longterm insulin treatment downregulated FOXO1 expression, while CRY1 deficiency rescued FOXO1 protein levels as well as gluconeogenic gene expression (Fig. 5c,d). It is of interest to note that CRY1-overexpressing mice showed a decrease of blood glucose level as well as of FOXO1 protein when AKT activity was pharmacologically repressed with AKT inhibitor MK2206 (Fig. 5g-i). Collectively, our in vitro and in vivo data suggest that the CRY1-dependent FOXO1 degradation would be one of the crucial mechanisms in attenuating hepatic gluconeogenesis for the long-term insulin action. Therefore, these observations prompted us to propose that the AKT-mediated FOXO1 phosphorylation provides an acute response during early insulin response, whereas SREBP1c-mediated CRY1 regulation would be a more durable process leading to the repression of futile hepatic gluconeogenesis throughout the anabolic state (Fig. 9).
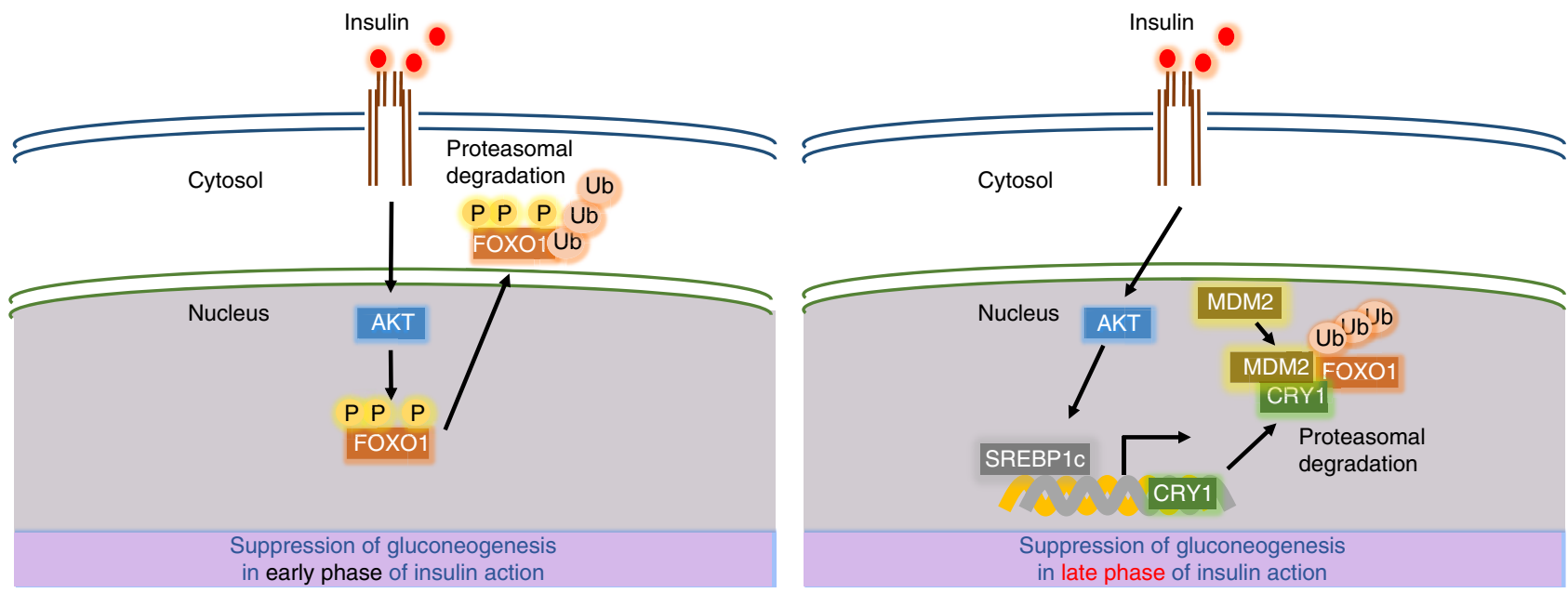

Figure 9 | SREBP1c-CRY1 axis suppresses hepatic gluconeogenesis by promoting FOX01 degradation. For long-term insulin action, SREBP1c-CRY1 axis suppresses hepatic gluconeogenesis through nuclear FOXO1 degradation. During anabolic state, increased CRY1 accelerates MDM2-mediated FOXO1 degradation and thereby insulin sustainably represses hepatic glucose production. 
As one of the key players for circadian rhythmic modulation, CRY1 expression is affected by various transcription factors and epigenetic regulation. Although our in vivo and in vitro data suggested that CRY1 expression was promoted by insulin and SREBP1c, CRY1 mRNA expression was not elevated by refeeding at ZT 10, which is the trough point of CRY1 oscillation (Supplementary Fig. 2B). These data propose that increased SREBP1c might not be sufficient to activate CRY1 at ZT 10. Conversely, the levels of CRY1 mRNA and proteins were robustly increased by refeeding at ZT 22, which is the peak point of its oscillation, implying that SREBP1c would augment CRY1 expression, probably, accompanied with other circadian regulatory factors. Nonetheless, it remains to be elucidated whether CRY1 as well as other circadian clock genes may contribute to modulate hepatic gluconeogenesis at ZT 22.

On the other hand, we asked the question whether SREBP1c deficiency would change hepatic circadian clock gene oscillation. As shown in Supplementary Fig. 12, hepatic circadian clock gene oscillations were not significantly different in SREBP $1 c^{+/+}$and $S R E B P 1 c^{-/-}$mice. Of course, we cannot exclude the possibility that SREBP1c-induced CRY1 might contribute to minor roles for hepatic circadian oscillation in SREBP $1 c^{-/-}$mice because it has been reported that $C R Y 1^{-/-}$mice exhibit fewer changes in circadian oscillations, compared with $C R Y 1^{-/-} C R Y 2^{-/-}$ double-mutant mice ${ }^{58}$. Furthermore, it is also possible that remaining SREBP1a and/or SREBP2 activity in SREBP1c ${ }^{-/-}$ mice might maintain intact circadian clock gene oscillations and this homoeostatic regulation needs to be addressed in future studies $^{21,59}$. Nonetheless, hepatic CRY1 gene expression is clearly upregulated by feeding/insulin-mediated SREBP1c.

Consistent with previous reports ${ }^{31}$, hepatic CRY1 was increased at night time (ZT 12-24) when feeding behaviour dominantly occurs in nocturnal animals (Supplementary Fig. 10A). Although $S R E B P 1 c^{-/-}$mice did not show any significant difference of circadian clock oscillation (Supplementary Fig. 12), SREBP1c expression was also increased at night time (ZT 12-24; Supplementary Fig. 10). Unlike the expression profiles of SREBP1c and CRY1, the level of FOXO1 protein appeared to be reduced at night time, implying that circadian oscillations of SREBP1c and CRY1 may contribute to repress diurnal glucose production by the regulation of FOXO1.

As SREBP1c could simultaneously regulate both gluconeogenesis and lipogenesis, it is plausible to speculate that hepatic SREBP1c would effectively coordinate the anabolic pathways by upregulating fatty acid metabolism and downregulating glucose metabolism on insulin signalling with different target genes. Our study is the first report to reveal the role of SREBP1c in CRY1 activation, which would be crucial in the regulation of hepatic glucose metabolism in the anabolic state. On the basis of the circadian oscillatory gene expression profile in $S R E B P 1 c^{-/-}$ mice, it appears that SREBP1c does not actively govern the hepatic circadian clock. Rather, increased expression of hepatic CRY1 during the postprandial state is primarily regulated, in a clock-gated manner, by insulin-activated SREBP1c, which eventually leads to the suppression of glucose production via FOXO1 degradation (Fig. 9). Although the roles of hepatic CRY1 in energy metabolism need to be investigated further, our data provide an important clue to understand the molecular mechanisms that link hepatic SREBP1c and glucose homoeostasis in physiological and pathological conditions.

\section{Methods}

Animals. C57BL/6 male mice were purchased from SAMTACO (Seoul, South Korea) and housed in colony cages. $d b /+$ and $d b / d b$ male mice were obtained from Central Lab (Seoul, Korea). SREBP1 $c^{-/-}$mice were generously provided from Dr J. Horton at the University of Texas Southwestern Medical Center and bred in isolated cages. All animals were maintained under $12 \mathrm{~h}$ light/12 h dark cycle in a pathogen-free animal facility. Following dissection, mouse tissue specimens were immediately stored at $-80^{\circ} \mathrm{C}$ until further analysis. All experiments with mice were approved by the Seoul National University Institutional Animal Care and Use Committee (SNUIACUC) and the Institutional Animal Care and Use Committee at the University of North Carolina.

In vivo imaging system. Ten-week-old $C 57 B L / 6$ male mice were injected with adenoviruses encoding green fluorescent protein (GFP) (Ad-MOCK), SREBP1c (Ad-SREBP1c) and G6Pase luciferase (Ad-G6Pase-luc) through the tail vein. After 5 days, adenovirus-infected mice were injected intraperitoneally with $100 \mathrm{mg} \mathrm{kg}^{-1}$ sterile firefly D-luciferin. After $5 \mathrm{~min}$, mice were anaesthetised and imaged using an IVIS 100 imaging system (Xenogen, Alameda, CA, USA) as described ${ }^{60}$.

Pyruvate tolerance tests. For the pyruvate tolerance test, mice were fasted for $16 \mathrm{~h}$ and then injected intraperitoneally with pyruvate $\left(2 \mathrm{~g} \mathrm{~kg}^{-1}\right.$ body weight for mice). Blood glucose levels were measured in tail vein blood samples at the indicated time points by using a Free-Style blood glucose metre (TheraSense, Sweden).

Materials and plasmids. MG132 was purchased from Calbiochem (San Diego, CA, USA). MK2206 was purchased from Selleckchem (S1078). AKTVIII was purchased from Santa Cruz Biotechnology (sc-202048). Antibodies against MYC (Cell Signalling, 2276, 1:1,000 dilution), HA (Cell Signalling, 3724, 1:1,000 dilution), FOXO1 (Cell Signalling, 2880, 1:1,000 dilution), phosphor-FOXO1Ser256 (Cell Signalling, 9461, 1:1,000 dilution), AKT (Cell Signalling, 9272, 1:1,000 dilution), and phosphor-AKT-Ser473 (Cell Signalling, 9271, 1:1,000 dilution), FLAG (Sigma-Aldrich, F3165, 1:1,000 dilution), ACTIN (Sigma-Aldrich, A5316, 1:2,000 dilution), G6Pase (Santa Cruz Biotechnology, sc-33839, 1:500 dilution), POLII (Santa Cruz Biotechnology, sc-899, 1:1,000 dilution), GFP (Santa Cruz Biotechnology, sc-9996, 1:1,000 dilution), GAPDH (LabFrontier, Co., LF-PA0018, 1:1,000 dilution), SREBP1 (BD Bioscience, 557036, 1:1,000 dilution), MDM2 (Abcam, ab16895, 1:1,000 dilution) and CRY1 (lab made antibody from Dr Aziz Sancar ${ }^{31}$, 1:200 dilution) were used. GFP-CRY1 was cloned into the pEGFP-N1 vector and FLAG-MDM2 was cloned into pCMV-3 FLAG. Mouse CRY1 promoter was cloned into the pGL3-basic vector.

Cell-based ubiquitination assays. COS- 1 cells (ATCC, CRL1650) were transfected with plasmids encoding FOXO1 WT-MYC, nFOXO1 (ADA)-MYC, GFP-CRY1 (or FLAG-CRY1), FLAG-MDM2 and Ubiquitin-HA in the presence of $20 \mu \mathrm{M}$ MG132 for $4 \mathrm{~h}$. Total cell lysates were prepared using the TGN buffer. FOXO1 WT-MYC and nFOXO1 (ADA)-MYC were immunoprecipitated with an anti-MYC antibody, and after washing in the TGN buffer, proteins were separated by SDS-PAGE followed by western blotting analyses with an anti-HA antibody.

ChIP assays. Cross-linking and chromatin immunoprecipitation assays with H4IIE cells (ATCC, CRL1548) were performed as described previously ${ }^{61}$. Extracted proteins from total cell lysates were immunoprecipitated with anti-SREBP1 (BD Bioscience) or IgG (Santa Cruz) for $2 \mathrm{~h}$. Precipitated DNA fragments were analysed by qRT-PCR using primer sets that encompassed the proximal $(-100$ to +100 base pairs) region of the rat CRY1 promoter and negative control $(+9670$ to +9890 base pairs) region. The sequences of ChIP assay primers were as follows: sense, $5^{\prime}$-GTCCGAGCCAGCGTAGTAAA-3' , antisense, 5' -GGATAGCGCGGG CTAGAG- $3^{\prime}$; negative control primer sense, $5^{\prime}$-CCAGCCACTTTGCTGAAGTT- $3^{\prime}$ and antisense, $5^{\prime}$-CTAGACAAGGCTGCCCACTC- $3^{\prime}$.

Preparation of recombinant adenovirus. The adenovirus plasmid was constructed as previously described ${ }^{62}$. Rat SREBP1c and mouse CRY1 cDNAs were incorporated into the AdTrack-CMV shuttle vector and a recombinant vector was generated using the Ad-Easy adenoviral vector system. Adenoviruses were amplified in HEK293A cells and isolated by caesium chloride density centrifugation. The GFP was co-expressed from an independent promoter with inserted cDNA. For in vivo experiments, mice were injected with $5 \times 10^{9}$ p.f.u. of adenovirus ( $d b / d b$ mice, $2 \times 10^{10}$ p.f.u.) in $200 \mu$ PBS through the tail vein. Empty virus expressing only the gene for GFP served as the control (MOCK).

Mouse primary hepatocytes cultures. Mouse primary hepatocytes were isolated from 10 -week-old male mice using collagenase perfusion method ${ }^{63}$ and plated with medium 199 (Invitrogen, Carlsbad, CA, USA). After $6 \mathrm{~h}$ of attachment, cells were infected with adenovirus or transfected with siRNA. For adenoviral infection, isolated hepatocytes were incubated for $4 \mathrm{~h}$ with adenovirus at $10 \mathrm{PFU}$ per cell with the serum-free medium, which was subsequently replaced by the $10 \%$ FBS-containing M199 medium.

Cell lysis and immunoprecipitation. After washing in cold PBS, cells were treated either with the TGN buffer ( $50 \mathrm{mM}$ Tris- $\mathrm{HCl}, \mathrm{pH} 7.5,150 \mathrm{mM} \mathrm{NaCl}, 1 \%$ Tween-20 
and $0.3 \%$ NP-40) or the SDS lysis buffer (200 mM Tris-HCl, pH 6.8, 10\% glycerol and $4 \%$ SDS) supplemented with $0.1 \%$ protease inhibitor cocktail (Roche, Basel, Switzerland). Total cell lysates were obtained by centrifugation at 12,000 r.p.m. for $15 \mathrm{~min}$ at $4{ }^{\circ} \mathrm{C}$, and $1-1.5 \mathrm{mg}$ of lysates was used for immunoprecipitation. The lysates were incubated with primary antibodies for $2 \mathrm{~h}$ at $4{ }^{\circ} \mathrm{C}$, followed by $1 \mathrm{~h}$ of further incubation with $50 \%$ slurry of protein A sepharose presaturated with the lysis buffer. After washing three times with the lysis buffer, the immunoprecipitated proteins were recovered from the beads by boiling for $10 \mathrm{~min}$ in the sample buffer and analysed by SDS-PAGE and immunoblotting.

Transient transfection and luciferase assays. HEK293T cells (ATCC, CRL3216) were transiently transfected with various DNA plasmids using the calciumphosphate method described previously ${ }^{64}$. After incubation for $36 \mathrm{~h}$, transfected cells were collected with the lysis buffer $(25 \mathrm{mM}$ Tris-phosphate $\mathrm{pH} 7.8,10 \%$ glycerol, $2 \mathrm{mM}$ EDTA, $2 \mathrm{mM}$ DTT and $1 \%$ Triton X-100) and the activities of luciferase and $\beta$-galactosidase were measured according to the manufacturer's protocol (Promega, Madison, WI, USA). The relative luciferase activity was normalized to $\beta$-galactosidase activity in each sample.

RNA preparation and quantitative reverse transcriptase-PCR analyses. RNA was prepared as previously described ${ }^{65}$. Briefly, total RNA was isolated using the TRIzol reagent (Invitrogen). Subsequently, equal amounts of RNA were subjected to cDNA synthesis using the RevertAid M-MuLV reverse transcriptase (Fermentas, Canada). The relative amount of mRNA was evaluated by using a CFX real-time quantitative PCR detection system (Bio-Rad Laboratories, Hercules, CA, USA) and calculated following normalization to the level of TBP or cyclophilin mRNA. The primer sequences used for the real-time quantitative PCR analyses are described in the Supplementary Table 1.

siRNA transfection. siRNA duplexes for CRY1, MDM2, and FOXO1 were purchased from the Bioneer, Inc. (Daejeon, South Korea). Primary hepatocytes were transiently transfected with the Lipofectamine RNAi MAX reagent (Invitrogen) according to the manufacturer's protocol. The sequence information for siRNA is described in the Supplementary Table 2.

Glucose production assays. Glucose production by mouse primary hepatocytes was measured according to the manufacturer's protocol using a glucose oxidase assay (Sigma-Aldrich, St Louis, MO, USA). Briefly, the cells were incubated for $6 \mathrm{~h}$ at $37^{\circ} \mathrm{C}$ and $5 \% \mathrm{CO}_{2}$ in the Krebs-Ringer buffer $(115 \mathrm{mM} \mathrm{NaCl}, 5.9 \mathrm{mM} \mathrm{KCl}$, $1.2 \mathrm{mM} \mathrm{MgCl}_{2}, 1.2 \mathrm{mM} \mathrm{NaH}_{2} \mathrm{PO}_{4}, 2.5 \mathrm{mM} \mathrm{CaCl}_{2}, 25 \mathrm{mM} \mathrm{NaHCO}_{3}, 10 \mathrm{mM}$ lactate and $2 \mathrm{mM}$ pyruvate, $\mathrm{pH} 7.4$ ). The glucose production assays were performed in triplicate.

Statistical analysis. Sample sizes were chosen based on pilot experiments that ensured adequate statistical power with similar variances. Statistical significance was assessed by the Student's $t$-test and are presented as the mean \pm s.d. determined from at least three independent experiments. Values of $P<0.05$ were considered to indicate statistically significant differences. All $n$ values defined in the legends refer to biological replicates unless otherwise indicated. If technical failures such as failure of intraperitoneal injection occurred, those samples were excluded from the final analysis. The experiments were not randomized. The investigators were not blinded to allocation during experiments and outcome assessment.

Data availability. The data that support the findings of this study are available from the corresponding author on reasonable request.

\section{References}

1. Summers, S. A. \& Birnbaum, M. J. A role for the serine/threonine kinase, Akt, in insulin-stimulated glucose uptake. Biochem. Soc. Trans. 25, 981-988 (1997).

2. Puigserver, P. et al. Insulin-regulated hepatic gluconeogenesis through FOXO1-PGC-1alpha interaction. Nature 423, 550-555 (2003).

3. Moller, D. E. New drug targets for type 2 diabetes and the metabolic syndrome. Nature 414, 821-827 (2001).

4. Wong, R. H. et al. A role of DNA-PK for the metabolic gene regulation in response to insulin. Cell 136, 1056-1072 (2009).

5. Wang, Y. et al. Phosphorylation and recruitment of BAF60c in chromatin remodeling for lipogenesis in response to insulin. Mol. Cell 49, 283-297 (2013).

6. Sugden, M. C., Caton, P. W. \& Holness, M. J. PPAR control: it's SIRTainly as easy as PGC. J. Endocrinol. 204, 93-104 (2010).

7. Mouchiroud, L., Eichner, L. J., Shaw, R. J. \& Auwerx, J. Transcriptional coregulators: fine-tuning metabolism. Cell Metab. 20, 26-40 (2014).

8. Yoon, J. C. et al. Control of hepatic gluconeogenesis through the transcriptional coactivator PGC-1. Nature 413, 131-138 (2001).

9. Accili, D. \& Arden, K. C. FoxOs at the crossroads of cellular metabolism, differentiation, and transformation. Cell 117, 421-426 (2004).
10. Kops, G. J. et al. Direct control of the Forkhead transcription factor AFX by protein kinase B. Nature 398, 630-634 (1999).

11. Lu, M. et al. Insulin regulates liver metabolism in vivo in the absence of hepatic Akt and Foxol. Nat. Med. 18, 388-395 (2012).

12. Matsumoto, M. \& Accili, D. All roads lead to FoxO. Cell Metab. 1, 215-216 (2005).

13. Tontonoz, P., Kim, J. B., Graves, R. A. \& Spiegelman, B. M. ADD1: a novel helix-loop-helix transcription factor associated with adipocyte determination and differentiation. Mol. Cell. Biol. 13, 4753-4759 (1993).

14. Brown, M. S. \& Goldstein, J. L. The SREBP pathway: regulation of cholesterol metabolism by proteolysis of a membrane-bound transcription factor. Cell $\mathbf{8 9}$, 331-340 (1997).

15. Yokoyama, C. et al. SREBP-1, a basic-helix-loop-helix-leucine zipper protein that controls transcription of the low density lipoprotein receptor gene. Cell $\mathbf{7 5}$, 187-197 (1993).

16. Moon, Y. A. et al. The Scap/SREBP pathway is essential for developing diabetic fatty liver and carbohydrate-induced hypertriglyceridemia in animals. Cell Metab. 15, 240-246 (2012).

17. Fajas, L. et al. Regulation of peroxisome proliferator-activated receptor gamma expression by adipocyte differentiation and determination factor $1 /$ sterol regulatory element binding protein 1 : implications for adipocyte differentiation and metabolism. Mol. Cell. Biol. 19, 5495-5503 (1999).

18. Peterson, T. R. et al. mTOR complex 1 regulates lipin 1 localization to control the SREBP pathway. Cell 146, 408-420 (2011).

19. Laplante, M. \& Sabatini, D. M. mTORC1 activates SREBP-1c and uncouples lipogenesis from gluconeogenesis. Proc. Natl Acad. Sci. USA 107, 3281-3282 (2010).

20. Kim, J. B. et al. Nutritional and insulin regulation of fatty acid synthetase and leptin gene expression through ADD1/SREBP1. J. Clin Invest. 101, 1-9 (1998).

21. Liang, G. et al. Diminished hepatic response to fasting/refeeding and liver $\mathrm{X}$ receptor agonists in mice with selective deficiency of sterol regulatory element-binding protein-1c. J. Biol. Chem. 277, 9520-9528 (2002).

22. Horton, J. D. et al. Combined analysis of oligonucleotide microarray data from transgenic and knockout mice identifies direct SREBP target genes. Proc. Natl Acad. Sci. USA 100, 12027-12032 (2003).

23. Ide, T. et al. SREBPs suppress IRS-2-mediated insulin signalling in the liver. Nat. Cell Biol. 6, 351-357 (2004)

24. Lee, Y. S. et al. Chromatin remodeling complex interacts with ADD1/SREBP1c to mediate insulin-dependent regulation of gene expression. Mol. Cell. Biol. 27, 438-452 (2007).

25. Becard, D. et al. Adenovirus-mediated overexpression of sterol regulatory element binding protein-1c mimics insulin effects on hepatic gene expression and glucose homeostasis in diabetic mice. Diabetes 50, 2425-2430 (2001).

26. Ponugoti, B., Fang, S. \& Kemper, J. K. Functional interaction of hepatic nuclear factor- 4 and peroxisome proliferator-activated receptor-gamma coactivator 1alpha in CYP7A1 regulation is inhibited by a key lipogenic activator, sterol regulatory element-binding protein-1c. Mol. Endocrinol. 21, 2698-2712 (2007).

27. Yamamoto, T. et al. SREBP-1 interacts with hepatocyte nuclear factor-4 alpha and interferes with PGC-1 recruitment to suppress hepatic gluconeogenic genes. J. Biol. Chem. 279, 12027-12035 (2004).

28. Miyamoto, Y. \& Sancar, A. Vitamin B2-based blue-light photoreceptors in the retinohypothalamic tract as the photoactive pigments for setting the circadian clock in mammals. Proc. Natl Acad. Sci. USA 95, 6097-6102 (1998).

29. Thresher, R. J. et al. Role of mouse cryptochrome blue-light photoreceptor in circadian photoresponses. Science 282, 1490-1494 (1998).

30. Vitaterna, M. H. et al. Differential regulation of mammalian period genes and circadian rhythmicity by cryptochromes 1 and 2. Proc. Natl Acad. Sci. USA 96, 12114-12119 (1999).

31. Ye, R. et al. Dual modes of CLOCK:BMAL1 inhibition mediated by Cryptochrome and Period proteins in the mammalian circadian clock. Genes Dev. 28, 1989-1998 (2014).

32. Green, C. B., Takahashi, J. S. \& Bass, J. The meter of metabolism. Cell 134, 728-742 (2008).

33. Zhang, E. E. et al. Cryptochrome mediates circadian regulation of cAMP signalling and hepatic gluconeogenesis. Nat. Med. 16, 1152-1156 (2010).

34. Lamia, K. A. et al. Cryptochromes mediate rhythmic repression of the glucocorticoid receptor. Nature 480, 552-556 (2011).

35. Hirota, T. et al. Identification of small molecule activators of cryptochrome. Science 337, 1094-1097 (2012).

36. Rudic, R. D. et al. BMAL1 and CLOCK, two essential components of the circadian clock, are involved in glucose homeostasis. PLoS Biol. 2, e377 (2004).

37. Jang, H. et al. Feeding period restriction alters the expression of peripheral circadian rhythm genes without changing body weight in mice. PLOS ONE 7, e49993 (2012).

38. Pilkis, S. J., el-Maghrabi, M. R. \& Claus, T. H. Hormonal regulation of hepatic gluconeogenesis and glycolysis. Ann. Rev. Biochem. 57, 755-783 (1988). 
39. Oh, K. J., Han, H. S., Kim, M. J. \& Koo, S. H. CREB and FoxO1: two transcription factors for the regulation of hepatic gluconeogenesis. BMB Rep. 46, 567-574 (2013).

40. McNeill, D. A., Herbein, J. H. \& Ritchey, S. J. Hepatic gluconeogenic enzymes, plasma insulin and glucagon response to magnesium deficiency and fasting. J. Nutr. 112, 736-743 (1982).

41. Kim, J. B. et al. Dual DNA binding specificity of ADD1/SREBP1 controlled by a single amino acid in the basic helix-loop-helix domain. Mol. Cell. Biol. 15, 2582-2588 (1995)

42. Griffin, M. J., Wong, R. H., Pandya, N. \& Sul, H. S. Direct interaction between USF and SREBP-1c mediates synergistic activation of the fatty-acid synthase promoter. J. Biol. Chem. 282, 5453-5467 (2007).

43. Rena, G., Guo, S., Cichy, S. C., Unterman, T. G. \& Cohen, P. Phosphorylation of the transcription factor forkhead family member FKHR by protein kinase B. J. Biol. Chem. 274, 17179-17183 (1999).

44. Matsuzaki, H., Daitoku, H., Hatta, M., Tanaka, K. \& Fukamizu, A. Insulin-induced phosphorylation of FKHR (Foxo1) targets to proteasomal degradation. Proc. Natl Acad. Sci. USA 100, 11285-11290 (2003).

45. Kato, S., Ding, J., Pisck, E., Jhala, U. S. \& Du, K. COP1 functions as a FoxO1 ubiquitin E3 ligase to regulate FoxO1-mediated gene expression. J. Biol. Chem. 283, 35464-35473 (2008).

46. Fu, W. et al. MDM2 acts downstream of p53 as an E3 ligase to promote FOXO ubiquitination and degradation. J. Biol. Chem. 284, 13987-14000 (2009).

47. Kakuma, T. et al. Leptin, troglitazone, and the expression of sterol regulatory element binding proteins in liver and pancreatic islets. Proc. Natl Acad. Sci. USA 97, 8536-8541 (2000).

48. Han, J. et al. The CREB coactivator CRTC2 controls hepatic lipid metabolism by regulating SREBP1. Nature 524, 243-246 (2015).

49. Yoon, Y. S. et al. Suppressor of MEK null (SMEK)/protein phosphatase 4 catalytic subunit (PP4C) is a key regulator of hepatic gluconeogenesis. Proc. Natl Acad. Sci. USA 107, 17704-17709 (2010).

50. Beaven, S. W. et al. Reciprocal regulation of hepatic and adipose lipogenesis by liver X receptors in obesity and insulin resistance. Cell Metab. 18, 106-117 (2013).

51. Chu, X. et al. Sterol regulatory element-binding protein-1c mediates increase of postprandial stearic acid, a potential target for improving insulin resistance, in hyperlipidemia. Diabetes 62, 561-571 (2013).

52. Ponugoti, B. et al. SIRT1 deacetylates and inhibits SREBP-1C activity in regulation of hepatic lipid metabolism. J. Biol. Chem. 285, 33959-33970 (2010).

53. Li, X., Monks, B., Ge, Q. \& Birnbaum, M. J. Akt/PKB regulates hepatic metabolism by directly inhibiting PGC-1alpha transcription coactivator. Nature 447, 1012-1016 (2007).

54. Gross, D. N., van den Heuvel, A. P. \& Birnbaum, M. J. The role of FoxO in the regulation of metabolism. Oncogene 27, 2320-2336 (2008).

55 . Kelly, M. A. et al. Circadian gene variants and susceptibility to type 2 diabetes: a pilot study. PLoS ONE 7, e32670 (2012).

56. Harding, A. H. et al. Polymorphisms in the gene encoding sterol regulatory element-binding factor-1c are associated with type 2 diabetes. Diabetologia 49, 2642-2648 (2006).

57. Zhao, X. et al. Multiple elements regulate nuclear/cytoplasmic shuttling of FOXO1: characterization of phosphorylation- and 14-3-3-dependent and -independent mechanisms. Biochem. J. 378, 839-849 (2004).

58. van der Horst, G. T. et al. Mammalian Cry1 and Cry2 are essential for maintenance of circadian rhythms. Nature 398, 627-630 (1999).

59. Im, S. S. et al. Sterol regulatory element binding protein la regulates hepatic fatty acid partitioning by activating acetyl coenzyme A carboxylase 2. Mol. Cell. Biol. 29, 4864-4872 (2009).
60. Lee, J. H. et al. Ring finger protein 20 regulates hepatic lipid metabolism through protein kinase A-dependent sterol regulatory element binding protein $1 \mathrm{c}$ degradation. Hepatology 60, 844-857 (2014).

61. Sakai, N. et al. Involvement of histone acetylation in ovarian steroid-induced decidualization of human endometrial stromal cells. J. Biol. Chem. 278, 16675-16682 (2003).

62. Lee, G. Y. et al. PIASy-mediated sumoylation of SREBP1c regulates hepatic lipid metabolism upon fasting signalling. Mol. Cell. Biol. 34, 926-938 (2014).

63. Jo, H. et al. Endoplasmic reticulum stress induces hepatic steatosis via increased expression of the hepatic very low-density lipoprotein receptor. Hepatology 57, 1366-1377 (2013).

64. Seo, J. B. et al. Activated liver X receptors stimulate adipocyte differentiation through induction of peroxisome proliferator-activated receptor gamma expression. Mol. Cell. Biol. 24, 3430-3444 (2004).

65. Kim, K. H. et al. Inhibitory effect of LXR activation on cell proliferation and cell cycle progression through lipogenic activity. J. Lipid Res. 51, 3425-3433 (2010).

\section{Acknowledgements}

This work was supported by the National Research Foundation of Korea (NRF) grant funded by the Korea government (Ministry of Science, ICT and Future Planning; 2011-0018312) to J.B.K. We thank the members of the Laboratory of Adipocyte and Metabolism Research for helpful discussion and Dr Jay Horton at the University of Texas Southwestern Medical Center for providing SREBP1c ${ }^{-/-}$mice. H.J., G.L., Y.G.J., and J.H.L. were supported by the BK21 program. The authors have declared that no conflict of interest exists.

\section{Author contributions}

H.J. designed and conducted the study, performed experiments, and wrote the manuscript. G.Y.L., C.P.S., G.L., Y.G.J., J.H.L., K.K.Y.C. and P.T. performed experiments and contributed to the writing of the manuscript. M.J.B., A.X. and A.S. designed and discussed the study and contributed to the writing of the manuscript. J.B.K. supervised the whole project, discussed the data, and edited the final manuscript. J.B.K. is the guarantor of this work and, as such, had full access to all the data in this study and takes responsibility for the integrity of the data and the accuracy of the data analysis.

\section{Additional information}

Supplementary Information accompanies this paper at http://www.nature.com/ naturecommunications

Competing financial interests: The authors declare no competing financial interests.

Reprints and permission information is available online at http://npg.nature.com/ reprintsandpermissions/

How to cite this article: Jang, H. et al. SREBP1c-CRY1 signalling represses hepatic glucose production by promoting FOXO1 degradation during refeeding. Nat. Commun. 7:12180 doi: 10.1038/ncomms12180 (2016).

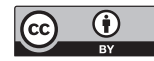

This work is licensed under a Creative Commons Attribution 4.0 International License. The images or other third party material in this article are included in the article's Creative Commons license, unless indicated otherwise in the credit line; if the material is not included under the Creative Commons license, users will need to obtain permission from the license holder to reproduce the material To view a copy of this license, visit http://creativecommons.org/licenses/by/4.0/

(C) The Author(s) 2016 